\title{
ÜBER DEN SYNTAKTISCHEN GEBRAUCH DES GENITIVS IN CYNEWULF'S ELENE, CRIST UND JULIANA.
}

So sehr auch in neuerer zeit und besonders seit uns durch Grein die denkmäler zugänglicher gemacht worden sind, das studium der angelsächsischen sprache vorgeschritten ist, so galten die untersuchungen doch meist der lehre vom worte, seiner bildung und flexion, während die lehre vom satze nur geringe beachtung gefunden hat. ${ }^{1}$ Ist die syntax einer sprache aber an sich schon wichtig und sind untersuchungen uber dieselbe notwendig, so ist dies im Angelsächsischen noch weit mehr der fall, da wir ihrer zur textkritik noch sehr bedürfen.

Selbstverständlich ist es aber auch, dass hier, wo fast noch gar nichts getan worden ist, zuerst mit einzeluntersuchungen anf einem begrenzten gebiete begonnen werden muss, bis es dann einer späteren zeit tuberlassen sein wird, diese untersuchungen zu sammeln, zu vergleichen und $z u$ dem ganzen einer angelsächsischen syntax zu verarbeiten.

Eine solche vorarbeit soll auch die vorliegende untersuchung tuber den syntaktischen gebrauch der casus bei Cynewulf - zunächst des genitivs ${ }^{2}$ - sein. Um einer späteren bekannt:

1 Von einzelabhandlungen über angelsächsische syntax ist mir nur

E. Nader, Der Nominativ, Genitiv und Accusativ im Beowulf. Drei programme der staatsoberrealschule zu Brïnn. 1879, 1880, 1882, und

Kress, Ueber den Gebrauch des Instr. in der angelsïchsischen Poesie. Göttingen 1865.

Ausserdem wurde noch eine angelsächsische syntax versucht in:

Koch, Historische Grammatik der englischen Sprache.

Mätzner, Englische Grammatik.

March, Anglo-Saxon Grammar.

${ }^{2}$ Die zusammenstellungen über den gebrauch des acc., dat. und instr. habe ich auch fertiggestellt und gedenke ich dieselben später zu veröffentlichen. 
zusammenarbeitung entgegenzukommen, bin ich dem vorgange Nader's gefolgt und habe mich in der einteilung, der hauptsache nach, an Erdmann angeschlossen. ${ }^{1}$

Da diese vorarbeiten firr später nur dann werden recht verwendet werden können, wenn in denselben therall vollständigkeit geboten wird, habe ich mich bemúht, möglichst vollständig zu sein.

Dass ich mich auf Cynewulf's Elene, Crist und Juliana beschränkt habe hat seinen grund darin, dass diese drei gedichte durch ihren vorwiegend geistlichen charakter eine gewisse zusammengehörigkeit bekunden. Obgleich Dietrich's ausfthrungen (in Haupt IX) mir durchaus nicht therzeugend waren, denn auf mich haben die hymnen nicht den eindruck eines zusammengehörigen ganzen gemacht, habe ich doch fur diese untersuchung - da seine behauptungen bis jetzt noch keine widerlegung gefunden haben - Dietrich's resultate angenommen und die unter dem namen Crist zusammengestellten hymnen als zusammengehöriges ganze behandelt.

Die idee, dass das woher, wohin und wo nicht erst durch verben den casus beigelegt sei, sondern gewissen casus urspriinglich innewohne, ist schon sehr alt. Schon bei den Byzantinern finden wir spuren davon. ${ }^{2} \mathrm{Zu}$ anfang unseres jahrhunderts, nachdem durch Humboldt die sprachwissensehaft in neue bahnen gelenkt worden war, wurde anch diese theorie wider mit grossem eifer aufgenommen. Dölecke, Wullner und Hartung bauten dieselbe weiter aus. Am besten ist diese fassung der lokalistischen theorie bei Hartung, welcher davon ausgieng, dass die wahrnehmung zuerst durch die sinne und dann durch den geist geschehe. Wie die sinnliche wahrnehmung der geistigen vorausgehen müsse, so wäre auch die sprache der ersteren zuerst dienstbar gewesen und dann erst wäre die sinnliche ausdrncksweise metaphorisch zur bezeichnung geistiger dinge verwendet worden. 'Aus den beziehungen nun in raume musste es sich ergeben, wie viele oblique casus von haus ans notwendig waren.'

10 . Erdmann, Untersuchungen iiber die Syntax der Sprache Otfrids. Halle 1874 (I. teil) und 1876 (II. teil). Fiir diese arbeit kommt nur der zweite teil: 'Syntax des Nomens' in betracht.

2 Vgl. Hübschmann, Zur Casuslehro. München 18i5. S. 22 u. 49. 
Indem nun Hartung dies weiter verfolgt und begrtindet, kommt er zu folgendem resultate:

1. Er findet für die bewegung und tätigkeit zwei casus :

a) den genitiv, als woher-casus;

b) den accusativ, als wohin-casus.

2. Für die ruhe nimmt er drei an:

a) instrumentalis (possessivus) oder wo-casus;

b) dativ, er driickt eine tendenz, das im angesicht liegende ziel, den afficierten oder beteiligten gegenstand, filr oder gegen den etwas geschieht aus;

c) ablativ, er dient zur bezeichnung des grundes.

Andere wider nehmen den ablativ als woher-casus und den dativ als wohin-casus an und bringen so ablativ und genitiv zusammen.

Gegen diese theorie kann man allerdings einwenden, dass es doch immer nur eine blosse annahme ist, zu behaupten, dass der mensch urspringlich nur sinnlicher wahrnehmungen fähig gewesen sei und dass ursprünglich die casus räumliche beziehungen ausgedrtickt hätten. Das wesentliche von Hartung's vorgange ist, dass er von den bedeutungen der casus ausgieng und mit diesen operierte, indem er von den tatsächlichen jetzigen casusverhältnissen seine behauptungen und aufstellungen abstrahierte.

So anschaulich nun diese theorie auch auf den ersten blick erscheint, denn es ist ja eigentlich selbstverständlich, dass verb und casus schon bevor sie in ein verhältniss zu einander eintreten, sich entsprechen, so hat diese ansicht doch entschiedene gegner gefunden; in neuerer zeit in G. Curtius. Dieser weist ${ }^{1}$ darauf hin, dass der einzig sichere ausgangspunkt hierftr nur die casusformen sein können. Er zeigt, nachdem er (s. 156) am nominativ nachgewiesen hat, dass hier die sprache von anderen als lokalen anschauungen ansgegangen sei, am accusativ die unmöglichkeit der lokaltheorie.

Der fehler der lokalisten war eben, dass sie mit den gegebenen casusverhältnissen rechneten und nicht mit den gewordenen, und dies muss deshalb schon geschehen, weil der nominativ und accusativ die einzigen casus sind, welche keine funktionen von anderen casus tbernommen haben, während genitiv und dativ als mischcasus auf uns gekommen sind. Im

1 G. Curtius, Erläuterungen zu meiner Schulgrammatik. Prag 1863. 
genitiv haben wir z. b. einen teil des fruh verloren gegangenen ablativs enthalten, wïhrend der andere teil vom instrumental absorbiert wurde und dann mit diesem an den dativ ubergieng. Daraus zu schliessen, dass der genitiv seiner bedeutung nach wirklich ein gleicher casus wie der ablativ sei, wäre naturlich ganz falsch, denn der reine genitiv erscheint eben nur als objektscasus. Wir dürfen dies um so weniger annehmen, als ja der genitiv beim verbum (vgl. s. 342) gar nicht ursprtinglich war, als solchen mutssen wir den genitiv beim nomen ansehen. Hier nun bezeichnet der genitiv die zusammengehörigkeit, während ja durch den ablativ gerade das gegenteil ausgedrickt wird. Dass sich, als das sprachgefthl sich verdunkelte, mit der zeit mittelglieder bildeten, darf uns nicht wundern, spricht aber noch nicht fur die ursprtingliche gleichheit der casus.

\section{Der genitiv.}

Ich werde zunächst den genitiv behandeln und folge hierbei, der hauptsache nach, der von Erdmann' ${ }^{\prime}$ und Nader ${ }^{1}$ angenommenen einteilung, indem ich immer Grimm's ' anordnung damit verglich, der fast in derselben weise verfahren ist.

Nach Grimm IV, 646 steht der genitiv dem accusativ in gewisser weise gegenther, indem er zwar wie letzterer auch ein casus des näheren objekts ist, aber nur eine 'geringere objektivisierung' zeigt. 'In ihm wird die tätige-kraft gleichsam dabei nur versucht und angehoben, nicht aber erschopft, während wir im accusativ die vollste, entschiedenste bewältigung eines gegenstandes durch den im verbum des satzsubjekts enthaltenen begriff haben.' 'In folge dessen kann man den genitiv auch nicht, wie den accusativ, in einen passiven nominativ umsetzen; während der accusativ reine, sichere wirkungen ausdruckt, finden wir beim genitiv gehemmte, modifcierte!' Wie das gebiet des accusativs ein grösseres und allgemeineres als das des genitivs war, so finden wir auch, dass die stellung des accusativs sich als eine gesichertere erwies, denn während in den neueren sprachen der accusativ eigentlich der einzige casus ist (ausser dem ihm oft formell gleichen nominativ), welcher sich noch rein, d.h. ohne präpositionelle

1 Erdmann a. a. o. $\S 173 \mathrm{ff}$.; Nader, Der Genitiv im Beowulf $\S 2$ ff; Grimm, Deutsche Grammatik. IV. teil. Syntax. Güttingen 1837. 
verdeutlichung erhalten hat, so gieng der genitiv und mit ihm auch der dativ als reiner casus verloren und wurden beide durch den casus obliquus mit entsprechender präposition ersetzt.

In bezug auf die entstehung des genitivs verweise ich auf Schrader ${ }^{1}$, welcher in anschluss an Höfer, Muller und Kuhn² den nachweis liefert, dass der genitiv ( $\gamma \varepsilon v \iota x i j \pi \tau \tilde{j} \sigma \iota)$ dem ursprunge nach vollständig sich mit dem adjektiv deckt; der bedeutung nach ist dies ja auch jetzt noch der fall, indem die art (das y'́vos), welche durch den genitiv ausgedrtickt wird, in allen sprachen ohne unterschied durch adjektiv mit substantiv (Schrader fuhrt den beweis mit avis aquatica und avis aquae) oder durch ein vom substantiv abhängiges substantiv im genitiv bezeichnet wird. Eine genauere untersuchung der betreffenden formen im Sanskrit, welches für solche fälle - da es die ältesten formen zeigt - für uns immer maassgebend ist, hat. auch zum resultat, dass die endungen des genitivs formell mit denen des adjektivs thereinstimmen. Curtius (Zur Chronologie der indog. Spr.) und Kuhn nehmen in folge dessen an, dass der genitiv - was Schrader fur sehr wahrscheinlich hält - nichts weiter sei als ein undeklinierbares adjektiv, doch lässt er die frage offen, ob nicht auch das gegenteil der fall sein könnte. ${ }^{3}$

Diese dem genitiv allein von allen casus eigenttimliche verwendung duirfen wir wol, nach dem zusammenhange mit adjektiven zu schliessen, als die ursprtingliche verwendung des genitivs ansehen 4 und hierdurch tritt dann der genitiv wider in gegensatz zum accusativ, indem bei ersterem das objekt attributiv steht, beim letzteren prädikativ. ${ }^{5}$

1 Sohrader, Ueber den syntaktischen Gebrauch des Genitivs im Gotischen. Gottingen 1874.

2 Hofer, Zur Lautlehre, Berlin 1839; M. Miller, Lectures on the science of language; Kuhn, Zeitschrift $X V$, s. 311 u. 425.

Als interessantes seitenstlick hierzu weise ich auf die entstehung unserer jetzigen possessivpronomina hin. Im Ags. - wie in den germanischen sprachen uberhapt - kannte man urspringlich die possessivpronomina nicht, als solche werden die genitive der personalpronomina verwendet. In der zeit unserer denkmäler nun finden wir die genitive zwar schon in adjektivischer verwendung, aber immer noch als genitive empfunden, während später dieses gefủhl vollständig schwand. Wir sehen also, dass hier die sprache einen entgegengesetzten weg - vom genitiv zum adjektiv - eingeschlagen hat.

4 Gl. dariber: Steinthal, Charakteristik s. 301; Curtius, Chronologie s. 256; Siecke, De genetivi in lingua sanscrita inprimis vedica usu. Berol. 1869; Htibschmann, Zur Casuslehre.

' Wie 'ich liebe den mann' und (weres frize) 'die liebe zum manne'. 
In den fällen, wo wir nun den genitiv im Angelsächsischen treffen, haben wir es nicht immer mit einem reinen, ursprunglichen genitiv zu tun; wie schon oben ${ }^{1}$ erwähnt, haben gewisse casus die funktionen anderer, verloren gegangener mit thernommen und so finden wir den genitiv zuweilen auch als vertreter eines alten ablativ, wobei er sich auch oft mit dem instrumental beruhrt und zwar so, dass genitiv und dativ-instrumental zugleich fur ein und dieselbe verwendung eines verloren gegangenen casus eintraten und wir nun zwei gleichberechtigte konstruktionen zur bezeichnung eines und desselben finden.2

Ich werde diese vertretungen an den betreffenden stellen noch besonders hervorheben und bin hierbei meist Delbrick ${ }^{3}$ und Hubschmann gefolgt.

\section{I.}

\section{Der genitiv in verbindung mit einem substantivum.}

§ 1. Wie schon erwähnt, ist dieser gebrauch - im Angelsächsischen wenigstens - nur dem genitiv eigentumlich. Für das Gotische bringt allerdings Schrader einige beispiele, in denen der dativ vom substantiv abhängig ist, doch stehen diese, mit noch einigen anderen ${ }^{5}$, ganz vereinzelt $d a$.

Die verbindung eines substantivs, in ganz beliebigem casus, mit einem abhängigen genitiv drtickt eine enge beziehung der durch die beiden substantive bezeichneten gegenstände insofern aus, als das regierende substantiv durch das hinzutretende, abhängige näher bestimmt wird. 6 Die art dieser beziehung oder bestimmung wird freilich durch das genitivverhältniss in keiner weise beleuchtet und bleibt es ganz der willktur tuberlassen, die gesichtspunkte - die allerdings meist äusserlicher natur sind - aufzustellen, nach denen man die einzelnen fälle von einander abheben kann und die uns dann einen gewissen therblick uber die art der verwendung des genitivs im besonderen gewähren. Nur zu diesem zwecke und um tberhaupt des reichen

1 Seite 340 und 341.

2 Z. b. wuldres full und wuldre zefylled oder ealdre linnan und ealdres linnan.

3 Delbriick, Ablativ, Locativ und Instrumentalis; Hübschmann, Zur Casuslehre.

- Schrader a. a. o. s. 12.

5 Grimm IV, 704.

- Vgl. Grimm IV, 71i; Erdmann a. a. o. § 173; Nader a. a. o. § 2. 
materials in gewisser weise herr zu werden, hat man solche gesichtspunkte aufgestellt. So sehr dieselben anfangs auch subjektiver art waren, hat man sich doch gewöhnt, die vorzüglichsten derselben allgemein anzuerkennen, und so babe ich denn kein bedenken getragen, denselben zu folgen.

Ich teile den genitiv beim nomen mit Erdmann (§ 173) 'nach der art, in welcher das im genitiv stehende substantiv aufgefasst ist', in einen individuell und einen generell gedachten genitiv.

\section{Individuell gedachter genitiv.}

$\S$ 2. Hier lasse ich znnächst das genitivverhältniss zweier personen folgen und setze an erste stelle den genitiv zur bezeichnung

a) eines verrwantschaftsverhältnisses, bezw. der abstammung. ${ }^{1}$

1. Vater.

yldra fæder El.437; yldra Salamônes El. 343; hâliz̧re fæder Jul. 61; pæ̂re fæ̂mnan fæder Jul. 67. 79. 159.

2. Sohn.

witzan sunu El. 592; monnes sunu Cr. 126.

Hier reihe ich nun gleich die vielen hierhergehörigen bezeichnungen firr Christus ein:

sunu meotudes El. 461. 474. 564. 686. 1318, Cr. 143. 197. 451.629; mæ̂re meotudes sunu Cr. 589; meotudes suna Cr. 94; mêrum meotudes suna Cr. 209; sunu wealdendes El. 892; s̀una waldendes Cr. 635; sunu sỗan fæder swezles Cr. 110; pinre sylfre sunu Cr. 339; sunu dryhtnes Cr. 345; zodes ẑ̧x̂stsunu El. 673, Cr. 660. 861; bearn zodes El. 814. 562. 837. 964. 1127, Jul. 666, Cr. 147. 774. 904. 1073; âzen bearn zodes El. 179. 422. 1077, Cr. 572; bearn êacen zodes Cr. 205; zodes êce bearn Cr. 744; bearn wealdendes El. 391. 851, Jul. 266; frêobearn zodes Cr. 643. 788; cyninzes frêobearn El. 672; meotudes bearn Cr. 126; sizebearn zodes El. 863. 481. 1147.

3. Andere verwantschaftsverhältnisse.

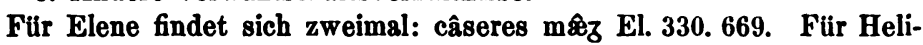
sêus einmal: hyre brydżnma Jul. 165. Für menschen (in bezug auf Adam und Eva): hyra eaferum Jul. 504.

b) Herrschaft.

Hierher gehören die zahlreichen, oft bildlichen ausdriicke fiir 'könig, herr'. Dass diese nicht gerade sehr zahlreich in diesen gedichten vertreten sind, um den first zu bezeichnen, hat seinen grund in dem vorwiegend geistlichen charakter der stücke, wir finden dieselben aber weiter unten, in grosser menge, auf Gott übertragen.

1 Nader \& 2 ff;; Grimm IV, 717. 
Die in Elene vorkommenden benennungen für könig und herr beziehen sich immer auf Konstantin, Jul. 66 ist Helisêus gemeint.

Einfach die herrschaft, den künig als solchen, bezeichnen baldor, bealdor (princeps), aldor, ealdor (princeps, dominus), hyrde (princeps, rex). Hildfruma (princeps bellicosus) und zâjweard (custos proelii) heisst der könig als erster und tïchtigster kämpfer, sowie als leiter der schlacht. Als beschützer seiner leute wird der könig helm (protector) und hleo (protector, tutor), weard (wart, wïchter, hiiter, beschützer, herr) genannt. Der künig ermutigte aber auch seine mannen zu neuen taten, indem er sie für die geleisteten dienste reichlich belohnte, er heisst in dieser eigenschaft bêazzifu (armillarum largitor, ringspender) und zoldwine (amicus aurum donans).

aldor secza El. 97; aldor folces El. 157. - baldor wîzena El. 344. rîces hyrde Jul. 66. -- hêrna hildfruma El. 101. - zû̉weard zumena El. 14. - herizea helm El. 148; weoruda helm hyrnwîzendra El. 223. æð̌linzes hlêo El. 99; eorla hlêo El. 1074; wìzena hlêo El. 150. - wîzena weard El. 153. - beorna bêazzifa El. 100. 1199. - zoldwine zumena El. 201.

Wie schon erwähnt, wird auch Gott in ähnlicher weise bezeichnet. Als könig und herr heisst Gott (und Christus): cyninz, drihten (princeps, dominus), hlaford (dominus, herus), pêoden (dominus), frêa (dominus), brezo (princeps), waldend (gubernator, rex, dominus). fruma (rex, princeps, procer) und ealdor. Als beschützer der menschen wird er genannt: weard, helm und hleo.

cyninz enzla El. 79, Cr. 715; cyninz Israhêla El. 800; hæleర̃ cyninz Cr. 372; cyninza ${ }^{2}$ cyninz Cr. 1682; ealra cyninza cyninz Jul. 289, Cr. 136. 215; mæzna cyninz Cr. 833; cyninz tre Cr. 494; hêahenzla cyninz Cr. 528; heofonenzla cyninz Cr. 1010; cyninz ælwihts Cr. 687; cyninz clênra zehwæs Cr. 703; weorodu wuldorcyninz Cr. 161. - duzuða dryhten El. 81, Cr. 782; weoroda dryhten El. 1140, Cr. 428; dryhten ealra hæleðs cynnes El. 187; dryhtna dryhten ${ }^{1}$ Jul. 593, Cr. 403; on hyrs dryhtne Cr. 1109. - hlâford ealra enzla and elda El. 475. - pêoden enzla El. 487. 777. 858, Cr. 332. 791. - enzla frêz El. 1307; frêz ealra zescesfta Cr. 925. - brezo enzla Jul. 666; hâliz hêohenzla . . Cr. 405. - mæzena waldend El. 347, Jul. 313; pêoda waldend El. 419. 781; weoroda waldend El. 752. 789. 1085, Cr. $15 \% 0$; waldend werpêoda Cr. 714; waldend enzla El. 773, Cr. 777; hyra waldend Cr.1186. 1214; ealles oferwaldend El.512, Cr. 544. 577; ealles waldend middanzeardes and mæzenprymmes Cr. 556; ríces ealles oferwaldend El. 1236. - herza fruma El. 210, Cr. 845; ealra folca fruman Cr. 516. - weoroda ealdor Cr. 229.2 - se Jâsta helm El. 176; wera helm El. 475, Cr. 634; hâližra helm Cr. 529. - żosta hlêo Jul. 49; beorna .. Jul. 272; wîzendra . . Cr. 409. — weard Israhêla El. 338; enzla weard El. 1101. 1316; folces weard Cr. 1648. - Elene wird einmal genannt: Cristenra cwên El. 1069, welches letztere wir auch in be-

Vgl. $\$ 9$.

Auch Juliana erhült einmal den beinamen fürst (bezw. fürstin) in: mæzða bealdor Jul. 568 .

Anglia, VIII. band. 
zeichnungen für Maria finden, sie heisst: mâre middanzeardes seo clæ̂neste cwên Cr. 275; seo clêneste cwến pârs pe Cr. 277. Im anschluss an diese benennungen heisst sie noch: hlæ̂fdize wuldorweorudes and worldcundra hâda and helwara Cr. 284.

Hieran schliesse ich noch drei beispiele an, in denen der teufel auch cyninz genannt wird:

hellwarena cyninz Jul. 322. 437. 544.

c) Nationalität (auch herrschaft, führerschaft).

Nur ans Elene, in welcher das verhältniss des herrn zu seinen leuten einige male berïhrt wird und wo sich überhaupt manches weltliche besonders kampf und kriegsfahrt betreffend - findet, kann ich beispiele zu diesem kapitel bringen.

Rômwara cyninz 62. 129; Hâna .. 32. 49. - Rôme bisceop 1052. Hûna lêode 20. 128. - weras Ebrêa 287. - Ebrêa pêod 448. - Hâna and Hrêta here 58. - Hûna herzes 143. - Judêa cynn 209. - Israhêla folc 361. - Israhêla æð̉elu 433.

d) Abhängigkeit, untertänigkeit.

Für dieses kapitel kann ich kein einziges beispiel aus dem wirklichen lehnswesen bringen, sämmtliche aus Juliana und Crist hier angefiihrten beispiele sind in uibertragener bedeutung gebraucht. Gott galt dem kriegerischen stamme der Angelsachsen vor allem als mächtiger 'könig' nnd volksherr' und in diesem sinne wurden auch die engel in 'degen und kämpen' umgewandelt.

zecorenan Cristes peznas Jul. 299; pâ hyhstan Cristes peznas Cr. 282; zodes pegnas Cr. 710. -- enzla zemanan Cr. 1046. - zodes cempan Jul. 17; modizne mẹtodes cempan Jul. 383; dêofla cempan Cr. 563. zodes enzel Jul. 261. 563, Cr. 315. - his bodan Cr. 1152; zodes .. Cr. 1305. - zodes spelbodan Cr. 336. - feonda zenirlan Cr. 1440.

e) Abstammung.

'Das regens ist ein collectivum oder ein plural (bearn); die verbindung streift an das formelhafte' (Nader $\S 2$, e).

lêoda bearn El. 181, Cr. 1159; lêoda bearnum Cr. 1425; ælda . . Cr. 937; fira .. Cr. 242; ealmanna bearn Cr. 85; dryzhtzumena .. Cr. 887. - ilda cynnes El. 521; fira .. El. 898; hæleঠa .. El. 1204; ofer eall wifa cynn Jul. 432; eal enzla cynn Jul. 640; m伊ða cynnes Jul. 550; fira cynne Jul. 509, Cr. 35; monna cynnes Jul. 470. 718, Cr. 957; ælda cynnes Jul.727, Cr. 786; zumena cynnes Jul. 718; wîzena .. Jul. 678; pêoda cynn Cr. 224; hæleða cynne Cr. 1197; eall monna cynn Cr. 888; Adames .. Cr.961. 1028; under helle .. Cr. 1620; Seraphinnes . . Cr. 386. - frumcyn fira Cr. 35. manna zecynd El. 735; woruldmanna seo clêneste zecynd Cr. 1016; eorбan zecynda Cr. 1181. - his æðelu Jul. 286; of hyra æర̈elum Cr.1181. -

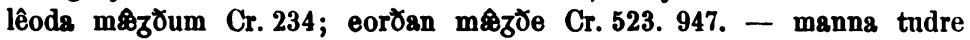
Jul. 459; monnes mazutudre Cr. 629. - wera cneorissum Cr. 1234. Cristes folces El. 499.

1 Vgl. die sub a angefthrten bezeichnungen und die weiteren benennungen in $\S 12$. 
§ 3. Zugehörigkeit einer person zu der in rede stehenden sache.

Hierfür habe ich nur ein beispiel aus Elene bringen können: holtes zehlêða 113.1

§ 4. Zugehörigkeit einer sache $z u$ der in rede stehenden person (poss. gen.).

Wie schon oben (s. 342, anm. 3) erwähnt, haben sich im Angelsächsischen die possessivpronomina aus dem genitiv der personalpronomina entwickelt. Ich fasse dieselben zusammen und fuge sie am ende dieser klasse an.

pêodnes burz Cr. 553; fêonda byriz Jul. 549; Cr. 569. - wrâđra wîc Cr. 1555. - dêaðsele dêofles Cr. 1537. - lindwîzendra land El. 270; on breca land El. 250. 262. 999; lifzendra londes Cr. 457; Cristes burzlond Cr. 51. - neorxna wanz El. $756^{2}$; neorxna wonzes Cr. 1391. 1406. enzla eard Cr. 646. - circe her æxfyllendra Cr. 703; zodes .. Cr. 699. enzla êđelstôl Cr. 52; fæder êđelstôl Cr. 516; zæ̂sta zifstôl Cr. 572. zodes tempel Cr. 707; torhtan tempel dryhtnes Cr. 180.

Körperteile: mâð æ̂nizes mannes El.660; purh pæs dêman mû̉ El. 1283; of mûðe mânfremmendra Cr. 1437. - pæs hêlentes heafelan Cr. 505. - sumra fêt Jul. 472; his frêan fêt Cr. 1169. - lîc hâliz̧re Jul. 689. - fæ̂̀mnan fæðm Cr. 788; enzla fæðmum Cr. 651.

Hierzu gehören auch:

zodherzendra hâlizra blôd ryht fremmendra Jul. 6. - ârlêasra spâtl Cr. 1436. - hæfta stefne pinnra niedpiowa Cr. 360 .

Leben: in wera life Cr. 416. - firs feorum Cr. 1593.

Handlungen, kampf': cwicra zewyrhtu Cr. 892. - weorcum Ebrêa Cr. 67. - monna dêde Cr. 1046. - cwicra zowin Cr. 998; zowinne dômeadizra Cr. 1656. - wið hettendra hildewôman Jul. 663. zrômra zârfare Cr. 781. - ealles mortorslehtes deareбlacendra deað̆ra El. 649. - æðelinzes plez̊ Jul. 743; hâlizes hyhtplez̊ Jul. 737. - zrômra zripe Jul. 391. - dêofla stræ̂las Cr. 779.

Besitz: Ârzestreon êtelcyninza Cr.997. - yrfes bracer wuldorcyninzes El. 1320.

Dann noch: æt zodes earce El. 392.

§ 5. Zugehörigkeit einer sache zu einer anderen.

temples hrôf Cr. 495. - cacernes duru Jul. 236. - pæs wealles zeat Jul. 401. - pæs ceasterhlides clustor Cr. 314. - pæs temples sezl Cr. 1139. - lifes zesteald Cr. 304. - heofones eac hêohzetimbro Cr.

- Die beispiele hierfür scheinen überhaupt sehr selten zu sein, denn auch Nader kann nur ein einziges, was noch dazu zweifelhaft ist, beibringen; vgl. Nader $\$ 4$. - Da holtes zehleja hier eine umschreibung fir 'wolf' ist, hat es ausserdem noch in $\$ 12$ einen platz gefunden. bringt.

' Vgl. hierzu die erklärung, welche Grein im 'Sprachschatz' s. 281

${ }^{3}$ Säınmtliche unter 'handlungen, kampf' angefilhrten verbindungen kijnnten ebenso gut zum gen. subj. (\$ 13a) gereohnet werden. 
1182. - eorơan scêatas Cr. 1005; ealne foldan scêat Cr. 72; foldan scêatum Cr.879. - purh ofnes fŷr El.1311. - mid lîchoman êazum Cr.1315. drync ecedes and zeallan Cr. 1439.

§ 6. Haften einer abstrakten eigenschaft an einer durch den genitiv ausgedruckten person oder sache.

purh zâstes mihtum El. 1070. 1100; ânes .. Cr. 567; meaht zodes Cr. 1625; pæs hyhstan .. Jul. 446; .. zoda ussa Jul. 614. - ânes cræfte

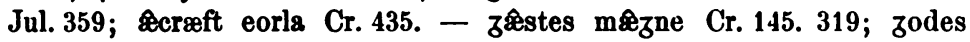

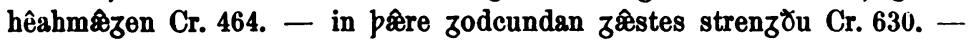
zâd friczendra El. 992. - pes pearf Jul. 717; . . môdes snyttro El. 553; pînra ârna .. Cr. 255; zâstes pearfe Cr. 707. 817. 1057. - purh drylitnes ઉâst El. 352. - wuldor pæs Cr.598. - heofones mæ̂rðu Cr.591. se enzla prym Cr. 1064; prijnisse prym Cr. 599; heofonduzuða .. Cr. 1655; heofons .. Cr. 653; heofonrîces .. Cr. 1634; eallum worulde prymmum Cr. 217; zod-prym êces alwaldan Cr. 139. - zeozuðhâdes blâd Jul. 168; his drêames .. Cr. 1587; peznna .. Cr. 1636. - êadižra blis Cr. 1650. - pâra ồerra êad Cr. 1294. - zeozuð̌hâdes zlêm El. 1267. wonzes beorhtne blêd welan Cr. 1392. - in cildes hâd El. 336. 776; Jldran .. Cr. 1669; fâmnan .. Cr. 92. - enzles hiw Jul. 244; monnes . . Cr. 657. - werzes blêo Cr. 1565. - ymb weres snyttro El. 959. -

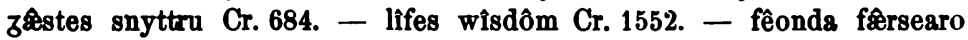
Cr. 770. - ædra wylm Jul. 477; in pæs lêades .. Jul. 583; ymb pæs wæteres .. El. 39; wæ̧zes .. Jul. 680; wonfŷres .. Cr. 965. - nerzendes noman El. 1165; in dryhtnes .. Cr. 413. - zodes miltsa Cr. 1686; miltse meotudes Cr. 1255. - æðelcyninzes wlite Cr. 907; beorht êঠles .. Cr. 1347; neorxna wonzes .. Cr. 1406; tô . . pæs hûses Cr. 1140. - wîfes zearnunz Cr. 40; sibbe enzla and monna Cr. 689; fore zodes sibbum Jul. 594. eardes uncyððu Jul. 701. - sefan monna Cr. 663. - sâwle sôðfæstra Cr. 53; sổfæstra sâwla Cr. 1687. - rîces tô bêacne Cr. 1066. - foretâcen feores Cr. 1556. - foldræste eardes Cr. 1029. - reordberendra

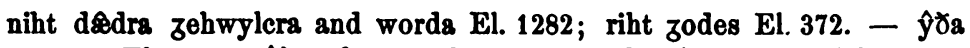
swenzas El. 239. - f̧ठa ofermets Cr. 855. - purh wêpnes spor Jul.623. wîzes spêd Cr. 673. - sốfæastra môd Jul. 325; pæ̂s mæżnes môd Jul. 608. - dryhtnes zemynd Cr. 1537. - incan womma zeworhtra Cr. 177. - pinzrêdenne môdzes zemanan Jul. 126. - pæs peznes.. môzroden Jul. 109. - w⿳⺈⿴囗十⺝⿱ wyrpan Cr. 565.

Hierzu rechne ich auch noch: on wênan deaðes, âdes and endeIffes El. 584.

In umgekehrter weise kann nun aber auch das abstractum im genitiv stehen; auch hierfir finden sich einige beispiele:

to wuldres byriz Jul. 665; .. ræste Cr. 1690. - sâwla rêst Cr. 1677. wommes tâcen Cr. 54. - pînra synna rôd Cr. 1490. - zesomninza sồtes and rihtes Cr. 700. - besludeda ælces nnrihtes Cr. 1303.

§ 7. Ich fuge jetzt die beispiele hinzu, in denen der genitiv eines possessivpronomens erscheint.

his rîce Jul. 8; on his cynestôle Cr. 1217; his hræżl Jul. 593; his frêan fêt Cr. 1169; his lichoman Cr. 1099; his môdor hrif Cr. 425; his dazena 
rim Cr. 1557; his wuldres miht El. 295. 727; his cræft and meaht Cr. 218; his ânes cræft Cr. 685 ; mid his wuldre Cr. 717; of his mæzenprymme Cr. 296; his drêames Cr. 1587; his weorces wlite Cr. 1588; his sâwle

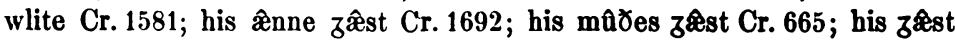
Jul.310, Cr. 1553. - mid hire burzum Cr. 968; hyre sâwle Jul. 669; hyre mæzdenhâd Cr. 1420. - hira dæ̂l El. 1232; mid hira fiscum Cr.967; hyra fiðrum Cr. 395; hyra dreorze liff Jul. 482; hyra lifes Cr. 1375; on hyra lîfdazum Cr. 1225; hyra weorcum Cr. 838. 1290; hyra ealdzestreon Cr. 1571; hyra blâd Cr. 1636; hyra swæ̂sne wlite Cr. 1149; hyra meaht andzefêa Cr. 107s; hyra drihtscipe El. 451; hyra sefan Cr. 1360; hira zôddênd El.369. - mînes fæder rîce Cr. 1345. - pîn sylfes weorc Cr. 9. sînra weorca wlite Cr. 103s. - usses dryhtnes rôd Cr. 1085. - êower hûs Jul. 648.

$\S 8$. 'In vielen fällen gibt der genitiv nicht eine eigensehaft an, sondern er bildet den hauptbegriff and das wort, von dem er abhängt, dient zu seiner erklärung.' 'Der inhalt des regierenden substantivums wird fur den in rede stehenden fall dem des im genitiv stehenden gleichgesetzt.' 'Es liegt nicht selten eine metapher vor.'1

Waffen: seaxes ecz Cr. 1141. - flana scâras El. 117. Uebertragen: se âttres ord Jul. 471, Cr. 768.

Schiff: cêoles bord Cr. 862 .

See: sæ̂s sîdne fæðm El. 729.

Feuer: fŷres fenz El. 1287. - fŷres blêo El. 1106. - ældes lêoma Cr. 1006. - purh pies fŷres fnæst Jul. 588. - in zlêda zripe El. 1302.

Brust: brêosta hord Cr. 1073; hreðerlocena hord Cr. 1056.

Land, erde: landes frætwe El. 1271; eorðan frætwan Cr. 106; feores frætwe Cr. 1074.

Sonne: sunnan lêoma Cr. 106. 901. - sunnan zyld Cr. 1103. - sunnan bryne Cr. 1662.

Auge: eazena lêoman Jul. 471.

Taufe: fulwihtes b. El. 490.1034.

Traum: swefnes wôma El. 71.

Posaune: heofonbŷman stefn Cr. 949; sîo bŷyman stefn!̣ Cr. 1062.

Sturm: storma scârum Jul. 651 .

Hieran kann man auch noch môd im sinne von mutig anschliessen, also: mannes môd Cr. $1691=$ der mutige mann.

§ 9. 'Durch derartige umschreibungen wird auch oft eine erhebung des begriffes erzielt; sie vertreten oft einen adjektivischen superlativ.'2

cræftes miht El. 585, Cr. 1146. - purh snyttru cræft El. 374, Cr. 667; mæznes cræfte Jul. 392. -- wîsdômes zewitt El. 357. 1191. - môdes snyttru Cr. 663. - ofer mæzena prym Cr. 757; wuldres .. Cr. 71. 83. -

1 Nader $\$ 7$.

2 Grimm IV, 723. 
heortan zehizdum El. 1224; heortan zehyzd Cr. 1039; heortan zehyzdas Cr. 1156; leahtra zehizdu Jul. 652, Cr. 1315. - môdes peaht Cr. 1242; heortan zepohtas Cr. 1048. - feores inzepanc Cr. 660. - hyzeponces fero El. 1332. - mid môdes myne Cr. 1359. - purh môdes zemynd Jul. 379. 657, Cr. 665. - frôfre 孔êst El. 1057. 1106. - wuldres wlite El. 311; ઉ̂िstes wlite Cr. 849. - mihta spêd El. 366, Cr. 296. 488. 1384; pầ miclan meahta .. Cr.652; æ̂hta .. El. 673; sâwla sizespêd El.1172. lîces lustas Jul. 409; earzes flêschoman ídelne lust Cr. 1298; firena lust Cr. 369; synna lustas Jul. 369; usse nioda lust Cr. 261. - leohtra firene Cr. 1281. - wom leohtra firena Cr. 1095. - pînra wîta bealo Jul. 211; helle bealu Cr. 1427; pŷystra .. Cr.1248. - se ezssan prêa Cr. 947. 1064. sinneahtes synnum Cr. 117. - môdes sorz Jul. 718; hyzesorze heortan mînre Cr.174. - synna wracu Cr. 1607; pŷstra wræce Cr. 593. - morðres mân El.626. - helle hienðu Cr.591. - prymmes præce Cr. 593. purh synna slide Jul. 349. - nî̉ heardra wîta Jul. 56. - môdes zælsa Jul. 336. - wôpes hrinz El. 1132, Cr. 357. - londes wynne Cr. 437; pæs eorð̌an .. Cr. 1667; leaslice líces .. Cr. 1297.

Von noch grősserer wirkung als die zusammenstellung zweier verwanter begriffe ist die verbindung eines substantivums mit seinem eigenen genitiv. Als hierhergehörend sind mir nur zwei beispiele vorgekommen: woruld weorulda El. 452; drêama drêam Cr. 580.

Dann aber kommt als umschreibung für den begriff Gott auch noch vor ealra prymma prym, lêohtes lêoht und cyninza cyninz, worauf ich an den betreffenden stellen schon aufmerksam gemacht habe.

$\S 10$. In ähnlicher weise werden zeitliche bestimmungen umschrieben:

on his dazans tîd El. 193; lifes .. El. 209; tîda dæzes Jul. 230; pæs sorza tîd Or.1572. - in pæs ĉrre lif êowres cynnes El. 305. - seofon nyhta fyrst El. 694. - syxte zeâr Constantines câserdômes El. 718. - zeteled rimes .. pinzemearces El. 3; dazens .. Cr. 467; his dazena rîm Cr. 1587. fram dæzes orde El. 140. - from fruman worulde Jul.509. - lifes æt ende El. 137; endo lifes El. 585, Jul. 661. - ઉeârs hwyrftum El. 1. zeâra zanzum El. 648, Jul. 693, Cr. 1036; wyrda .. El. 1256. - wyrda bizanz El. 1124; tîda . . Cr. 235. - in dazum Maximinianes Jul. 2; se micla dæz meshtan dryhtnes Cr. 869; on pam zrimman dæze dômes pæs miclan Cr. 1205; on pam dæze pæs ælmihtizan Cr. 1372. - nihtes nearwe El. 1240. - In ähnlicher weise steht auch: ealdordôm uncres zewinnes Jul. 190.

§ 11. In gleieher weise werden anch lokale bestimmungen durch das genitivverhältniss ansgedrtickt und finden sich hier sehr zahlreiche und verschiedenartige umschreibungen fur himmel, holle, erde und meer. ${ }^{1}$

H im mel: rodores ryne El. 795; .. tungla Jul.498, Cr.671. - tunzla zonz Cr. 884. - heofona zehlidu Cr. 518. - heofona zehyld Cr. 545. -

Vgl. hierzu auch $\S 12$. 
swezles hlêo El.507, Cr. 606. - ofer wolena hrôf El. 89; rodores.. Cr. 60. - rodera ymbhwearft Jul. 113.

Hölle: helle zrund El. 1315, Cr. 265. - helle sear Jul. 422.

Erde: wonza bizonz Cr.680. - zemetu middanzeardes Cr. 827. - burza zesetu Cr. 1240. - eorðan rices Cr. 880.

Me er: holma bizonz Jul.112. - ymb pæs wæteres stæర El.60. - zeofenes stream El. 1201. - sês sîdne fæðm El. 729.

§ 12. Heinzel (Ueber den Stil der altgerm. Poesie) fuhrt anf s. $18 \mathrm{f}$. eine ausdrucksweise an, durch welche vorstellnngen, 'die den alten Germanen besonders wertvoll, lieb oder grossartig erschienen', umschrieben wurden. Diese oft kthnen und edlen bilder lassen uns den gegenstand der umschreibung erraten, im gegensatze zu den in den letzten paragraphen behandelten umschreibungen, welche denselben selbst mitnannten and zugleich einen teil seines wesens - wie or fur den zusammenhang in den betreffenden stellen gerade am passendsten schien - hervorhoben. Diese umschreibungen fthren den namen Kenningar und finden sich in unseren gedichten besonders zahlreich für den begriff der gottheit, mit denen ich auch beginne.

Umschrieben wird der begriff der gottheit ausser durch die schon erwähnten benennungen cyninz, waldend, dryhten, fruma, ealdor, frêa, hyrde, weard, helm, hlêo, noch durch folgende: Allgemein zur bezeichnung der gottheit dient zod (deus, numen). Als schöpfer heisst Gott: meotud (schöpfer, der messende), scyppend (creator). Als helfer, heiland, retter: nerzend (salvator), zecocend (auxiliator, salvator), hâlend (salvator, heiland, spez. für Christus). Als herr wird Gott genannt: beoden (dominus), azend (possessor, dominus). Als richter, beim jungsten gericht, hoisst er: demend (judex). Als fihrer: latteon (dux).

Von den eigenschaften Gottes sind folgende benennungen genommen. Von seiner guite, als verleiher von glilck und seligkeit: sellend (dator, largitor), brytta (largitor, dispensator, administrator), wuldor zifa (verleiher von herrlichkeit), wil zifa (freudengeber, könig), hyhtzifa (dator largitiae), zoldhort (thesaurus). - Von seiner weisheit: rêniend (das rechte anordnend) von rènian (disponere, instituere, ordinare, praeparare, moliri). Dann heisst er noch: cedelinz (nobilis), prym (msjestas, magnificentia, pompa), wuldor (gloria, glorie, ruhm, herrlichkeit, preis), leoht (lux), lêome (lumen, splendor), ord (initium, nobilissimus, princeps), zôst (spiritus, sanctus), foeder.

wuldres cyninz El. 565, Jul. 516; heofones .. El. 61. 482; heofona .. El. 1009. - sizora dryhten El. 346. 1140; hâlza heofona .. Cr. 348. pêoden enzla El. 487. 777. 858. - sizora frêa Ed. 488; số sizores.. Cr. 404; sizora frêan Jul. 361; heofona hêahfrêa Cr. 253. 424. - rodera waldend El. 206. 452. 1067, Jul. 305, Cr. 866; wuldres .. El. 1090; wyrda . . 
El. 80; mihta . . El. 357. 1043, Jul. 723, Cr. 823; sizora . . El. 732; heofones . C Cr. 555. - lifes fruma El. 793, Cr. 44; leohtra .. El. 839; sizores .. Cr. 294; fyrnweorca .. Cr. 579; lifes ordfruma Cr. 22i; êades .. Cr. 1199; æðelne ordfruman ealra zesceafta Cr. 402 ; torhtes tîrfruman Cr. 266. - wuldres ealdor Jul. 153; prymmes .. Jul. 448. - wuldres helm Cr. 463; heofons .. Jul. 722; heofonrices .. Cr. 566. - heofonrîces weard El. 197. 416. 718, Jul. 212; rodera . . Cr. 134. 222; lifes . . Cr. 1642; se sâwle .. Cr."1551; sizores . . Cr. 243; sizora . . Cr. 1517; wuldre . . Cr. 527. - prymmes hyrde El. 348. 859, Jul. 280. - meotod moncynnes Jul. 182. 436. 667, Cr. 244; mæzencyninza . . Cr. 943. - scippend ealra El. 371; ઉâsta .. El. 791, Jul. 181; hæleða scyppende Cr. 266; moncynnes milde scyppend Cr. 147; hyra scyppend Cr. 1132; from his scyppende Cr. 1618. - Zâsta zêocend El. 682. 107T, Cr. 198. - nî̀a nerzend El. 503. 1086; . . firz El. 1078. 1174, Jul. 240; folca .. Cr. 426; sâwla . . Cr. 571. - liffes âzend Jul. 223. - dêda demend Jul. 725. - lîfes lâttêow El. 899. - sizora sellend Jul. 668. 7115. -- boldes brytta El. 162. weoruda wuldorzifa El. 681. - weoruda wilzifa El. 815. - hæleða hyhtzifa El. 852. - mæżena zoldhord Cr. 787. - rihtes rêniend El. 880. wuldres æðelinz Cr. 158. - cyninza prym El. 816; wìzena .. El. 1090; ealra prymma ..' El. 483, Cr. 726; rodera .. Cr. 423; wuldres .. Jul. 641, Cr. 740. - cyninza wuldor El. 5. 178, Jul. 279; beorna .. El. 181. sôffæstra lêoht El. 7; ealles leohtes lêoht ${ }^{1}$ El. 485; wuldres .. Cr.1673. ểles lêoma El. 1294; sốfæestra sunnan lêoma Cr. 695. - æð̌elinzes ord El. 393, Cr. 515. 741. 846. - frôfre ż̂st Jul. 724, Cr. 207; wîdômes . . Jul. 516; swezles .. Cr. 203. - fæder enzla Jul. 274. 784; fæðer ûser Jul. 545; sôđan .. swezles Cr. 110; . . frumsceafta Cr. 472; . . frôfre zâst Cr. 728; .. frêoð̃a Cr. 773. - frumð̄a zod El. 342. 582; eallra prymma .. El. 519; heofona .. El. 1125, Jul. 239; hêahenzla .. El. 751; sizora . . El. 1308; mihts . . El. 786. 819; mæzena .. El. 810, Jul. 109. 729; heofonmæzena . . Cr. 1218; weoruda .. El. 1150, Jul. 515, Cr. 347. 407. 631; zæ̂sta .. Cr. 130; mid zode líðes lîfes Cr. 1637. - wuldres zod Jul. 180.

Während die hier angefulhrten umschreibungen auch für Christus mit verwant werden, so findet sich noch nur für ihn: hælend middanzeardes El. 809 - eallre sybbe bearn Cr. 446 - hêafodhealle mêre Cr. 4. Ferner: sunu Dauîdes Cr. 712 - Jacobes bearn Cr. 164. Für Maria: Dauîdes dohtor Cr. 191 - Dauides dyrre mêzan Cr.96. His dohtor Cr.91 wird für die bewohnerinnen von Jerusalem gebraucht und in kühner wendung wird Juliana seo wuldres mổ Jul. 600 genannt.

In ähnlicher weise finden sich auch umschreibungen für 'teufel, götze' u. s. w.

his zodu Jul. 598; his zodum Jul. 252. - fêond moncynnes Jul. 317. 523. 630; sâwla.. Jul. 348; his ealdfêondum Cr. 567. - synna fruman Jul. 362; fyrnsynna.. Jul. 347; eallie synne fruma El. 772. - helle dêofol Jul. 629. - helle hæftlinz Jul. 246. - pŷstra stihtend Jul. 419. - hêan helle z êst Jul. 457.615. - wrôhtes wyrhtan Jul. 346. - wuldres wiðer-

Vgl. hiorzu §9. 
breca Jul. 269. - zleaw zyrnstafa zæstzonîঠla Jul. 245. - zodes andsacan Cr. 1594. - hæleða zewinna Jul. 243; hæleða zewinnan Jul. 345. fêonda forespreca Cr. 753. - se wites bona Cr. 204. - moròres mânfrea El. 942, Jul. 5ł6. - mânes melda Jul. 557, El. 428. - firena bearn Cr. 1556. - synna bryttan El. 958.

'Himmel' wird einmal umschrieben mit wuldres eard Cr. 1203.

Hieran schliesse ich noch einige umschreibungen für 'engel' und die 'untertanen des teufels'.

Engel: wuldres âras Cr. 493. - heofones hêahenzel Cr. 202.

'Teufel: sûsles peznum Jul. 558; pam wyrrestan wites .. Jul. 152. unclệnum dêofla zâsstum El. 302.

Für 'könig' finden sich - nur in Elene - folgende umschreibungen:

w⿳⺈⿴囗十⺝ hes helm 230 - sinces brytta 194 - hira wilzifan 1132.

Kampf: borda zebrec El. 114. - beorna zeprec El. 114. - herza zrinz

El. 115. - wîzes woma El. 19. - wôlhrêowra wìz El. 112. - wið̌

pêoda præce El. 185.

Besonders zahlreich sind die umschreibungen flir 'kreuz' in Elene: sizores tâcen 85. 184. 1121; heofoncyninzes .. 171; buton zodes tâcne Jul. 491. - bêacen zodes El. 109. - wuldres bêam El. 217; rodorcyninzes .. 839; wuldres wynbêam 887. - wuldres trêo 206; lifes trêo 664. 706. 757. 1027; rôde . . 206, Jul. 417. - Cristes rôde El. 103; æð̋e]cyninzes rôd 219.886; se rôd radorcyninzes El. 624.

Hölle: purh pæs dômes fŷr El. 1304; wîtes fŷr Cr. 625. - in dracan fæס̀me El. 766. - sûsla hûs Cr. 1604. - êరel enzla dreames Cr. 1343. - synna sêaঠ் Jul. 413. - in sûsla zrund El. 944. - pæs wilmes zrund El.1229. - lîzes locan Jul. 474, Cr. 1621. - in wita forwyrd El. 765. - under womma scêatum El. 583.

Tod: dêà̃es bend Cr. 1042. - deorc dêàes scêsdu Cr. 118.

Einmal für 'torriegel': homra zeweorc Jul. 237. Fur 'wolf' findet sich einmal in Elene: holtes zehlêða 113.

Ich schliesse dieses kapitel mit einer zusammenstellung der benennungen fur Juliana und Maria.'

Juliana: mînra êazena lêoht Jul. 95. - sunnan scîma Jul. 166. wlitescyne wuldres condel Jul. 454. - wizena wynn Jul. 641. æðelinza . . Jul. 730.

Maria: wîfa wynn Cr. 71. - brŷd pæs sêlestan swezles bryttan Cr. 280.

§ 13. In gewissen substantiven, die eine handlung oder einen vorgang bezeichnen, ist noch so viel verbalsinn, dass sie mit den von ihnen abhängigen genitiven bald aktive, bald passive bedeutung haben können. Nur der zusammenhang des satzes oder der gebrauch zeigt uns hier die art der verwendung (ob als genitiv subjectivus oder genitiv objectivus) an.

1 Vgl. fuir Maria $\S 2$. 
a) Genitivus subjectivus.

purh weres frize El. 341; pæs weres .. Jul. 103; weres frizum Cr. 37; weres friza Cr.419. - lêofra lufu Cr. 1653; pæs beornes lufan Jul. 41. hâlizra hyze Jul. 339. - fæ̂mnan foreponc Jul. 227. - wîszewit witzan dryhtnes Cr. 1193. - hâlizra hyht Jul.642. - his forzifnesse Cr.427. Cristenra zefêan El. 980. - meotudes êst El.986. - purh dêofles spild El. 1119. - feondes zenî̀lan El. 701. - pêoda zebæ̂ru El. 659; beornes .. El. 710. - dryhtnes willa El. 1160, Jul. 602; willa beza zehwæðeres El. 964; willan pêodnes El. 267, Cr. 1236; zodes .. Jul. 365, Cr. 1582; wîfes .. El. 1132; mid hyre fæder .. Jul. 32; meotudes . . Cr. 1236; sylfes willum Cr.1484. - pôre fêmnan word Jul. 59; pæs lârrêows word Cr.458; cyninzes .. Cr. 1630; word zodes Cr. 1204; pæs enzles .. Cr. 824; witzena .. Cr. 469; be pînes bonan worde Cr. 1394; cyninzes .. Cr. 1627. monizfealdra mæzna zerŷno Cr. 603; dryhtnes zerŷne Cr. 41. - enzla sonz Cr. 1650; witzena woðsonz Cr. 46. - peodnes zehâta Jul. 541. cwâniendra cirm Cr. 836. - purh pæs hâlz̧an hæ̂s El. 86. -- dryhtnes bibod Cr. 1159; heofoncyninzes .. Cr. 1525; beorht bôca .. Cr. 1631. fædera lâre El. 388; lâre witzena El. 335; mildan meotudes .. Cr. 1211); Saules lârum El.497; lêahtr̊ frumsn lârum El. 839; lâttêowes.. El.1210. -

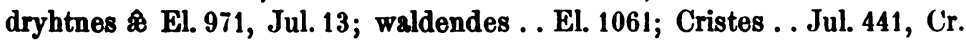

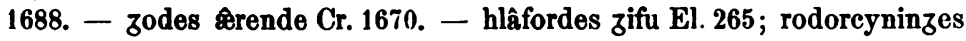
ziefe Jul. 447; cyninzes . . Cr. 1663; his . . Cr. 682. - wundor zodes El. 1122. - zempescipe monnes Cr. 199. - Euan scyld Cr.98. - moncynnes mânforwyrhtum Cr. 1095. - pæs brổan synwræce Cr. 793. synna wunde El. 514, Jul. 710, Cr. 1314; synna wundum Jul. 355. woruldwidles wom Cr. 1007; .. Êrran wunde Cr. 1322; wizes womum Jul. 576. - pæs unrihtes andsæc Cr. 472. - fêonda zefær El. 68. - werodes bearhtme El. 39; herizes .. El. 205. - pæs fuzles flyht Cr. 639. 654. - pæs dêman yrre Jul. 256. - hæleða ræ̂das El. 156; beza ræ̂dum El. 1009; hæleðð zer仓̂dum El. 1054. 1108. - purh finzra zeweald El. 120; on fêonda.. Jul. 159, Cr.1416; under hæঠonra hyrda zewealdum Cr. 705. wyrma slite Cr. 1251. - idese sîðfæt El. 229; his sîðfæt Jul. 285. - sunnan wilsîð Cr. 26. - rodorcyninzes ræ̂s Cr.727. - his upstize Cr.615; .. êcan dryhtnes Cr. 711. - Hnña cyme El. 41; pâr̊ næzla .. El. 1086; sumeres .. El. 1228; lêohtes .. Jul. 161; beza .. hwîtra and sweartra Cr. 897; purh pæs beornes .. Cr. 530; waldendes .. mæzen cyninzes Cr. 916; Cristes .. Cr. 1031; his hyhstan hiðercyme Cr.141. - pîn selfes zonz Cr. 254. - in pæs æঠelcyninzes æhtum Jul. 37.

b) Genitivus objectivus.

for sâwla lufan El. 564; lufan dryhtnes El. 491. 948. 1206, Jul. 501; fore Cristes lufan Jul. 31; lêohtra .. Jul. 375; for ælda .. Cr. 1117; for monna . . Cr. 1434. - liffes hyht Cr. 585. - eslles lêohtes zefêa Cr.585; êcne zefêan wuldres pînes Cr. 159. - zodes ezsa Jul. 35; fæder ezsan Cr. 1014. Cristes lof El. 212, Jul. 233; heofoncyninzes .. El. 748; zodes .. Jul. 408. 693; from Cristes lofe Jul. 139; of . . pînre êadzife Jul. 275. — pâra bealudß̊de bôte El. 515; blindnesse bôte El. 389. - æt pæ̂re zesyhðe pæs sizebêames ze pæs zelêafan El. 965; êazena zesihðe Cr. 7. 1114. fore onsŷne êces dêman Cr. 837. 796; sêo dyre dryhtnos onsien Cr. 1651; 
zodes .. Cr. 480; Cristes .. Cr. 906; onsŷne êcan dryhtnes Cr. 395. zodspolles ziefe Fl. 176; ż̂stes .. El. 199. 1058. 1157, Jul. 316; Cr. 649. 710; wîsdômes .. El. 596. 1114; hôlo . . Cr. 374. - wîzzes lêan El. 825;

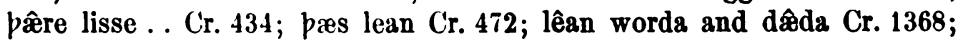
wuldres .. Cr. 1588; liffes tô lêane Jul. 708; wundorlêan worca Cr. 1080. dryhtnes prowinze Cr. 1175. - wâ zehwæð̀res El. 628. - in wîta for-

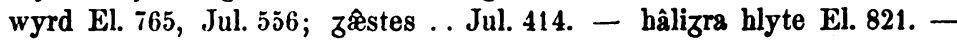
wið̀ hunzres hlêo El. 616. - wîra zespon El.1135. - tô beadwe burzwîzendra El. 34. - dôma zeweald El. i26; dômes .. Cr. 228; ealra .. Cr. 1648. - pæs unrihtes andsæc El. 472. - Cristes dolz Cr. 1207. foldan zesceafte Cr. 953. - lifes word Cr. 1393; word frâfre Cr. 1512. êacnunze bearnes Cr. 75. - Cristes zebyrd Cr. 65; bearnes .. Cr. 38. 788. - zodwebba cyst Cr. 1135; cyst cynestola Cr. 51; folzoða cyst Cr. 390. - mæzða weolman Cr. 445.

\section{Generell gedachter genitiv.}

\section{$\S 14$. Genitivus partitivus.}

'Der genit. part. ist ein genitiv der zusammengehörigkeit mit einem ganzen oder, wie man es ftr viele fälle richtiger ausgedrickt hat, mit einer gesammtheit.' 1 Er findet anwendung 'bei angaben der zahl und des maasses, oder bei individueller andeutung bestimmter gegenstände durch pronomina'. Dann steht anch bei angaben, welche durch den superlativ von adjektiven gegeben werden, die bezeichnung des allgemeinen, welchem der gezählte, gemessene, hervorgehobene gegenstand selbst mitangehört, im genitiv. Ich werde zuerst diesen genitiv in seiner abhängigkeit von einem substantivum behandeln.

a) Substantivum.

worn worda, sîdra sorza ... sârewida, hearmes Cr. 169. - lifwynna dôl Cr. 80ī; ânizne .. pŷstra Cr. 1385. - zedwolens rim Jul. 36s; his dazena .. Cr. 1587; dazena rîmes Cr. 467. - beorna unrîm Jul. 469; hyrsta .. Jul. 43; bisza . . Jul. 625; wita . . Jul. 17I; folces . . Cr. 569. enz̧la prêat Cr. 738; heofonenzla .. Cr. 492. 928; pezna prêate El. 151; beorna .. El. 873; wìzena .. El. 217; secza .. El. 271; zumena prêate El. 254; zumena zodherzendra .. El. 1096; on wera . . El. 537; scaðena.. Jul. 672; folca .. El. 215. - on wera corore El. 304. 543; êadizra zedryht El. 1290, Cr. 1664; enzla .. Cr. 942. 515. 1014; pezna .. Cr. 457; folca . . El. 27; folcdryht wera Cr. 1067. - halizra weorud Cr. 1649; synfulra .. Cr. 1229; wæ̂rleosra .. Cr. 1614; zlædzumena .. Cr. 1654; enzla weorude El. 1281. - eorla menzu El. 225; wera .. El. 596, Jul. 509, Cr. 45. 509. - pezna hêap El. 549, Cr. 944; lêofra hêap El. 1206; hellII, $\S 190$.

1 Curtius, Erläuterungen su meiner Schulgrammatik, s.164; Erdmann 
warena . . Cr. 731. - pâra synfulra sâwla fề an Cr. 1519. - heofonenzla here Cr. 1278; fềonda .. Cr. 1626; synfulra .. Cr. 1533; herzas hâlizra Cr. 930. - hêahenzla mæzen Cr. 1019; . . werze monna cynnes Cr. 957. secża hlôte Jul. 676. - ælbeorhtra sceolu Cr. 929; womfulra .. Cr. 1535; scyldizra .. Cr. 1608; byrnendra scole Cr. 1252. - on clæ̂nra zemanz El. 96, Jul. 420; on fềonda .. El. 108; on zramra .. El. 118. - folc zodes Cr. 764. - onhâlo zelâc enz̧la and deofla, bêorhtra and blâcra Cr. 895.

b) Nach zahlwörtern.

âna ealra monna Cr. 287; on hira x̂nne Cr.1172; on ânre niht Cr.626. pâra rôda twâ El. 880; on .. halfa El. 955; on .. halfe El. 1180. - on prêo healfa Cr. 1268; prêo .. rôda El. 833. - siex tìda dæzes Jul. 230. on sêofon healfa Cr. 950. - prittiz and fêowere eac .... wîzena cynnes Jul. 680. - fêowertiz dazena rîmes er. 446. - fif and hund sêofontiz hêðnes herzes Jul. 588. - tâ hund and prêo zeteled rîmes swylce prittiz pinz̧emêarces wintra El. 2 ff. - fifhund forðsnottera lêodmæ̂za El. 380. - pûsend manna El. 326; prêo pûsend pêra lêoda El. 285.

c) Genitiv nach quantitativen adverbien.

Diese adverbia sind aus substantiven entstanden und können auch noch als solche betrachtet werden.'

pæs wanzes wiht El. 184; wiht forholen monna zehizda Cr. 1054; and sîo weres friz̊ wiht ne cûđe Cr.419. - Ôwiht swylces El.571; âr pon ôht pisse æfre wurde Cr. 230. - yfles nôht² Jul. 238.

fels. Es ist als nominativ zu fassen in: is nu fela forðzewitenra and zôdra, zlêawra zumena El. 636; pêr wîsna fela wearo inhîhted Cr. 43. - Als accusativ ist fela zu nehmen in: feala wundra zefremede El. 362; nefre he feala sôtra wundra zefremede El. 779; feala mê se hôlend hearma zefremedo níða nearolícra El. 912 f.; pus ic wrâtra fela .... bêalwa zefremede ... sweartra synna .... heardra heteponca Jul. 311-15; ne wîta pæs fela .... wrâđra zezearwaঠ Jul. 177; pæt pu êr fela unwærlicra worda zespræ̂ce Jul. 192; and me hosp sprecað tornworda fela Cr. 172; pæt him tâcna fela onwrâh Cr. 462; feala dêadra worde âwehte El. 945; pæt he firenfremmendra fela prowade Cr. 1118; pâ ic zôda swa fela forziefen hæfde Cr. 1400; feala môla beĥेded El. 987.

Zweifelhaft; da das verb fehlt, ist der casus von fela in: feala tîda El. 1044. - tô fela: pæet hy him yrmða tô fela seoð Cr. 1269; mâzon wêana tô fela zeseon Cr. 1264; Ic tô fela hæbbe pæs brydscipes bealwa onfonzen Cr. 181. - eal-fela: se mæz eal-fela sinzan and seczan Cr. 666.

Mit einer präposition verbunden erscheint fela in : and mid wîta fela frêcnum feorhzomum folcum scendeð Cr. 1548.

mâ: pær bì wundra mâ El. 989; yfeldæda mâ.

tô lyt: hæfde wîzena tô lyt eaxlzestealna .... hrôra tô hilde El.63-65; êades tô lyt Cr. 1401.

1 Grimm IV, 727 fasst wiht (got. vaihts, ahd. wiht) noch als substantivum auf, auch im Ags. haben wir es noch. als subst. mit allen casus erhalten. Vgl. Grein, Sprachschatz s. 703 f.: wiht, wuht, wyht f. n. = creature, animal, res, wesen.

2 nôwiht. 
lythwon: lythwon... Huna herzes El. 142.

fea: pêah hira fea wæron El. 14.

zenôze: zenôze atol earfoða ârzedênra Cr. 1265.

$\S 15$. Partitiver genitiv bei unbestimmten numeralien und pronomen.

a) maniz: monize Crîstes folces El. 499; pæt he manizum wearð folca tô frofre El. 501. — æniz: æ̂niz yldra oððe zinzra El. 159; . . elda Cr. 311; fira æ̂niz Jul. 218; æ̂niz ælda cynnes Cr. 780; ஷ̂̉iz pâra

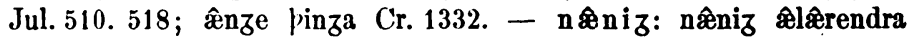
ổer betera El. 506. - nân: hêohfædera nân ne witzena Jul. 514; ne pæs miclan mæzenprymmes nân Cr. 351 .

b) sum. 'Wenn sum auf den gen. pl. von zahlwörtern oder von adj., die vielheit und wenigheit bezeichnen, folgt, so driickt es den begriff der begleitung aus.' (Grimm IV, 458): feara sum Cr. 12i6. sum kann aber auch attributiv - als adjectivum - mit einem substantiv verbunden werden: sum wôðbora Cr. 302.

c) hwylc: hwylc hyra Cr. 398; on hwylcum pâra bêama El. 851; on hwylcre pysse prêora El. 858. - zehwylc: pinza zehwyle El.409, Jul. 224; alra tâcna .. El.645; ac pe firina zewhyle feor âbûzeঠ̆, werzðo and zewinnes Cr. 56; wâżdeora .. Cr. 988; monns .. Cr. 589. 1051; zumena.. Cr. 821; ofer mæzna zehwylc Jul. 222; folc ânra.. El. 1287, Cr. 1026; mâna .. El. 1317; ânrs .. Cr.1030; ofer wîd landa.. Cr. 1385; zehwylces tâcna El. 319; eafota zehwylces El. 423; mâna.. Jul. 730; yfla zehwylces Jul. 352; wîta zehwylces El. 1030; womma. . El. 1310; zêasne zoda zehwylces Jul. 216; pinza . . El. 1156; rihta.. feohzestreona El.910; sizora .. Jul. 224; monna zehwylcum Cr. 431; zehwylcum zumena El. 278; folca .. Cr. 1219; pêoda zehwylcre Cr. 848; zehwylcre firena Cr. 180; fêonda zehwylcne El. 1179; scylda dêopra firena .. El. 1313; lêahtra .. Cr. 1309; zehwylce dêda Cr. 525; sâwla .. Cr. 1068; wihta .. Cr. 982; pêoda .. Cr. 1024. - zehwâa: niða zehwâm El. 465; үâra monna .. El. 1229, Jul. 728; beorna .. El. 1187; lifzendra zehwâm Cr. 231; leoda .. Cr. 193; dôzora .. Cr. 428; on sylfra .. Cr. 1242; worda zehwæs El. 569; yfla .. Jul. 323; sizora .. Jul. 562; se pe reorda . . ryne zemiclsठ Cr. 47; zehwone monna zumena cynnes Jul. 718; lêofra . . Cr. 816; moncynnes .. Cr. 1027; mânwomma .. Cr. 1281); tîda zehwane Cr. 107; ymb healfa ze-

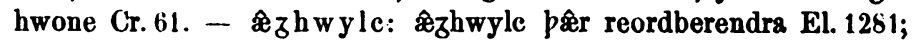
ख̧̂̉hwylcum synwyrcendra Cr. 841. - nâthw ylc: nâhthwylc hæleða

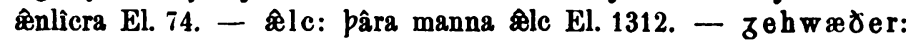
beza zehwæঠ̀res willa El.964. - pæt: pæt wæs pâra pinza Cr.224. -

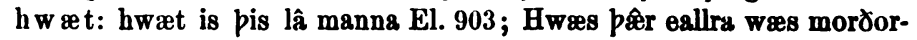
slehtes deareðlâcendra dêadra zefallen Cr. 649; zôdes hwæt Jul. 397; hwæt .... micelra mânweorca Cr. 458.

$\S 16$. Der partitive genitiv beim comparativ und beim superlativ.

Fiir den comparativ sind die beispiele sehr spärlich, ich kann nur drei sichere anführen: 
sunnan beorhtra El. 1110, Cr. 1242. - stâne heardran El. 365. - sunnan leohtre Cr. 1652.

Wenn man mîn als genitiv anffassen darf, was Grimm (IV, 735, 754) leugnet, auch Nader nimmt es als possessivum an, so wiirde noch: yldra mîn El. 462 hinzuzufügen sein.

Ungleich zahlreicher nun sind die beispiele für den superlativ, die ich hier anfige:

frôfra mêst El. 196. 993; znornsorza .. El. 977; wilspella . . El. 984; symbla .. Cr. 550; foretâcna . . Cr. 893; swêżdynna . . Cr. 955; morðorhûsa . . Cr. 1625; wôlfŷra . . Cr. 932; bæ̂lfîra .. Jul. 5i9; se bið cwealma .., dêofla and monna Cr. 1627; beaduprêata . . El. 31; fyrda . . El. 35; weo-

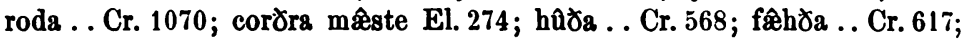
prymma .. Cr. 837; bearhtma . . Cr. 951; sorza . . Cr. 1082. 1200; scoma. . Cr. 1274. - sêlest sizebêacna El. 975; sêlest sizebêama El.1029; sêlust sizelêana El. 527; sêleste hæleða cynnes El. 1204; sêleste mid Judêum zumena El. 1202; ealra sizebearna pæt sêleste and æঠ̀eleste Cr. 520. æðelust bearna El. 476; æðelust tunzla Cr. 607; æðelast eorðan zecynda Cr. 1181; pâm æð̌̀lstan eorðcyninz̊ burzâzendra El. 1174. - burz̧a betlicast Cr. 66. - zefêana fæzrast Cr. 1665 . - weoruda wlitescŷnast Cr. 1665. - se hŷhsta ealra Cr. 1681. - tâcna teorhtost El. 164. lêohts beorhtost El. 948; enzla . . Cr. 104; bêacna .. Cr. 1086. - monna lêofast Jul. 84. - dêorast ealra Jul. 697. - woða wlitezaste El. 749. mârost beama El. 1013. 1225. - ج̂rest zesceafta Cr. 1153. - lâsta sîðast Jul. 474. - mircast mânweorca Jul. 505. - hâttost heaðowelma El. 509. wyrda lầost El. 978. - blâtast benna Cr. 771. — Jêsta zifrast Cr. 814. daza ezeslicast Cr. 1202.

II.

Der genitiv bei verben.

§ 17. Die verbindung eines verbums mit einem davon abhängigen genitiv hat in den meisten fällen partitiven charakter. ${ }^{\prime}$ Sie ist, wie schon im eingange bemerkt wurde, von allgemeinerer bedeutung und bedarf, wenn die begrenzung eine schärfere werden soll, noch einer näheren bestimmung durch einen anderen casus oder durch das hinzutreten einer präpositionsverbindung. Diese genitive können sowol persönlich sein, als auch, was meistens der fall ist, sächlich. Das 'unbestimmte etwas', welches nach Hubschmann und Nader ${ }^{2}$ der genitiv von einer person oder sache anssagt, nimmt bei den persönlichen genitiven doch eine etwas schärfere, bestimmtere gestalt an. Wenn man nun - wie s. 342 angefthrt wurde - die adnominale verwendung des genitivs als die ursprtingliche ansieht, so muss man sich

Vgl. Grimm IV, 650.654. 657.

2 Vgl. Hubschmann III. B; Nader $§ 18$. 
fragen, wo beim genitiv nach verben der nominalbegriff sei. Erdmann (s. 154 ff.) stellt dafür zwei erklärungen auf. Einmal, meint er, könnte man das subjekt des satzes als solchen betrachten und den genitiv als prädikative bestimmung dazu auffassen, wofür dann auch der genitiv bei wesan und weorठan spräche, der ja auch mit dem attributiven gebrauche des genitivs eng zusammenhängt, dann aber hält er es nicht für unmöglich, 'dass die handlung selbst, als ihr eigenes objekt mitgedacht', den nominalbegriff' gebildet habe. Diese auffassung wird uns wahrscheinlich gemacht durch verbindungen wie andswarian neben andsware cyðan, von denen ein genitiv abhängt. Sicher lässt sich dies jedoch nicht mehr entscheiden.

Schon im eingange dieser untersuchung habe ich hervorgehoben, dass wir im genitiv einen sogenannten mischcasus haben, da von ihm auch zum teil funktionen des ablativs und instrumentals aufgenommen wurden. Ich habe diese etymologisch verschiedenen genitive zu trennen gesucht nnd behandle den genitiv als vicariierenden casus an besonderer stelle.

1. Zunächst betrachte ich den ursprünglichen genitiv, der uns a) bei den verben der bewegung und des strebens erscheint. Ich beginne mit

nêosan $=$ visere, visitare, adire. Der aufgesuchte gegenstand steht im genitiv: cwôm pâ wízena hlêo pezna prêate pryơ bord stênan, beadurôf cyninz, burza nêosan El. 152; zesâwon wuldres prym, æðેelinza ord êđles nêosan Cr. 741; pŷstra nêosan Jul. 554; fêond moncynnes onzan pâ on flêam sceacen wîta nêosan Jul. 631. Der weg, den man dabei zurücklegt und die bertihrten örtlichkeiten werden durch purh in verbindung mit einem substantivum ausgedrtickt: zod wille .... purh pâ frestan locu foldan nêosan Cr. 321 .

êhtan -- persequi, tribulare, affligere wird mit dem genitiv der verfolgten person konstruiert: se êhteð pîn El. 928; .... êhton elpêoda El. 139.

tilizan = sludere, niti, intendere hat den gen. der erstrebten sache nach sich. In unserem beispiele finden wir das mittel des strebens doppelt ansgedriickt: einmal durch mid mit instr. und dann durch purh unit acc. Beide präpositionen vertreten einen früheren instrumental: mid hâ micle elne $\hat{x} z h w y l c$ wille purh ealle list lifes tilizan. El. 1318.

zesyrwan = rüsten, armare, machinari wird ausser dem gen. der person mit dem instr. des mittels verbunden: $p \hat{y}$ ic wide ferz sweortra zesyrede Jul. 467.

$\zeta \mathrm{ef} \hat{\mathrm{s}} \mathrm{an}=$ accelerare, properare, promptum abeundi reddere hat den gegenstand oder die sache, zu denen man bereit ist, im gen. bei sich: wâron æ̂xscwiżan, seczas ymb sizecwên, sîtes zefŷsde El. 260. 
Die person, der man widersteht, wird bei

wiostandan $=$ widerstehen, einem gegenüber stand hallen, durch den dat., die sache, in der man widersteht, durch den gen. und das mittel durch die präpos. purh ausgedriickt: pe pe oft wiðstôd purh wuldorcyninz willan pines Jul. 427.

biddan = petere, poscere, precari, deprecari, rogare, postulare hat die sache, um die man bittet, im gen.: huru pæs biddað burzsittende Cr. 337 .

Eine eigentïmliche konstruktion findet sich in folgendem satze: ponne hy him purh mînne noman êaðmôde tô êow ârna tâdon Cr. 1352.

Die person, welche etwas erbittet, steht im nom. und die person, für welche etwas erbeten wird, im dat. (eth.), der erbetene gegenstand im gen. und die person, von welcher etwas erbeten wird, wird durch to mit dat. ausgedriickt. purh in purh mînne noman (= in meinem namen) entspricht unserem 'bei' in 'jemand bitten (beschwören etc.) bei'. Statt tô mit dat. sollte man hier eigentlich den acc. erwarten.

b) Die verben des wartens, hütens, waltens werden mit einem sïchlichen gen. verbunden:

bîdsn = exspectare: cêolas lêton bîdan beorna zepinzes El. 253; nn̂ hîe sôfte pæs bidon in bendum Cr. 147; bîdað̉ hêofiende beorhte zesceafte dryhtnes dômes Cr.1021. = consequi, nancisci, sustinere: sêo circe eahtnisse bâd Cr. 704 .

c) Verba der empfindung und wahrnehmung:

cunnian = probare, tentare, explorare, experiri, perclitari, adire hat die erprobte sache im gen. bei sich stehen: swâ se fâla fuzel flyzes cunnode C'r. 645.

Bei zêcwêman = satisfacere, morem gerere, servire steht neben einem sächlichen gen. oft ein dat. der person: pôr ic swiðe me pyslîcre âr praze ne zecwêmde Jul. 452.

Ebenso verhält es sich mit âprêotan = taedere, pigere: êow pæs lungre âprêat $\mathrm{El} 368$.

d) Verba der geistestätigkeit:

zepencan = bedenken, beherzigen hat die sache, die man bedenkt, im gen.: âr sceal zepencan zôstes pearfe se pe Cr. 1057. = recordari, memor esse, gedenken: and usse yrmða zepenc Cr. 370; in zemynd habban: Ic pæs wuldres trêowes oft, nales æxene, hæfde, inzemynd El. 1252.

wề $\mathrm{nan}=$ wahnen, hoffen, erwarlen, worauf rechnen, sich eines dinges versehen: ne we pêre wyrde wênan purfon tôweard in tîde Cr. 81; pêr sceolon pêofas and pêoðsceaðan lêase and forlizene lifes ne wênan Cr. 1611; hwôr ne pâra næżla swî̀ost on pam wanzstede wênan porfte El. 1104; Hwæs wênað se Cr. 1200; ârna ne wênað Cr. 1232; lîfes ne lissa zeswênan Cr. 1611; Ne porftan pâ peznas in pâm pŷystran hâm seo zenêatscolu in pam neolan scræfe tô pam frumzare feohzestealdra witedra wênan Jul. 686 .

Mit reflexivem dativ (fir sich etwas hoffen) findet sich wênan verbunden in: wênde him praze hnâzre El. 668. In dem satze: rîces ne wênde for werodlêste tritt for zu wênan. for (fore) wird häufig fiir einen instr. gebraucht, um die ursache zu bezeichnen. 
ऊîman = curam habere, curare, observare, custodire: Ic pஷ̂re sâwle mâ zeornor ẑỳme ... . ponne pæs lichoman Jul. 414; hlâfes ne zime El. 616; pæt hio mâzllufan mînre ne ẑิye, frêondrêdenne Jul. 70; pæ̂r pâ synsceaðan sôðes ne ziemdon, ż̂stes pearfe Cr. 706; zrundlêase ziemeঠ̀ zâsta on pêostre Cr. 1546; ponne pæs zìman nelle weoruda waldend C'r. 1569.

myndzan = in memoriam revocare, monere, suggerere, memor esse: we pæt hereweorces hlæfdize mîn for ${ }^{1}$ nyjdpêarfe nean myndza El. 652. Unpersünlich und mit acc. der person findet es sich gebraucht in: Mec pâra næzla zen on fyrhðsefan fyrwet myndzað El. 1078.

aðolian = nobilitare: feores forhtlîce forð aðolian Cr. 132.

e) Verba der rede und mitteilung:

seçan = sagen, sprechen. Das gesagte steht im gen.: pæs pe æ̂fre sundbûend seczan hŷrdon Cr. 73.

mânian = ermahnen, mahnen, monere, suggerere, hortari, reducere in memoriam rei. Die sache, an welche man ormahnt wird, steht im gen.: pæs lifes ic mânize Cr. 1479.

pancjan = danken, zeigt den dativ der person, welcher man dankt; wofiir man dankt wird durch den gen. ansgedrickt: ponne he $p \hat{y}$ zeornor zode panciað blædes and blissa Cr. 1256.

Hieran reihe ich gleich verba wie dank sagen, welche den acc. 'dank' als inneres objekt bei sich haben und mit diesem einen begriff bilden, von dem dann, wie bei 'danken' ein gen. abhängt. Jedoch kann man diese verbindungen auch so auffassen, dass man 'sagen' als ein den acc. erforderndes verb annimmt und von dem acc. 'dank' dann den gen. abhïngig macht:

ponc seczan: sæzde ealles ponc dryhtna dryhtne Jul. 503.

ponc cunnan = dank wissen: pâm pe ponc zode womwyrcende wita ne câton Cr. 1092; hy pæs êtles ponc hyra waldende wita ne cuton Cr. 1213.

ponc witan = dank wissen: pæt pu waldende pinre âlŷsnesse ponc ne wisses Cr. 1473 ; pâ pu pæs ealles mnizne ponc pînum nerzende nysses on môde Cr. 1498.

In allen diesen beispielen findet sich noch ein dativ der person, welcher man dankt.

çेðฉn = nuntiare, anuntiare, referre, indicare, enuntiare, effari, praedicare. Neben dem gen. der sache, welche man verkindet, steht hier ein dativ der person, zu der man redet: Ic pe, êad môz, yfla zehwylces ôr zecŷðe ơ ende forঠ Jul. 352.

In gleicher weise wie ponc seczan etc. kann man auch andsware c $\hat{y} \gamma_{a n}=$ untworten als einen begriff auffassen, von dem der gen. abhängt: pầ me sôflice andsware cŷỹan for êow for tâcna zehwylces El. 318.

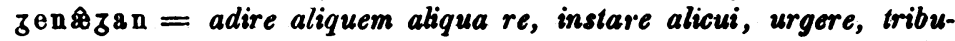
lare, appelare findet sich mit acc. der person und gen. der sache: eorlas unzearwe yfles zenæ̂geð Cr. 875.

1 for- vgl. s. 360 .

Anglia, VIII. band. 
Bei friczan = interrogare, soscitari steht das, was man erfragt, im gen.: ðâ pæs friczan onzan folces aldor ofer sî̉ weorod El. 157.

forwyrnan = recusare, denuere, denegare hat die person, der etwas versagt wird, im dativ bei sich; das, was jemandem versagt wird, steht im gen.: se pe ổrum forwyrneð wlitizan wilsî̀es Cr. 20.

Wird dann noch das mittel angegeben, durch welches die verweigerung bewirkt wird, so geschieht dies durch purh mit einem substantivum: me hwîlum bið forwyrned purh wiðerzesteall willan mînes hyhtes æt hâlzum Jul. 440.

f) helpan = kelfen mit gen. der person, der man hilft: ponne ze hira hulpon Cr. 1354; pæt ze earmra hulpen Cr. 1503.

g) Die verben des gebens, empfangens, gebrauchens und geniessens.

Einen dativ der person, der etwas geschenkt wird, mit dem genitiv der geschenkten sache zeigt unnan $=$ gonnen, gewahren, verleihen, schenken: zen ic feores pe unnan wille Jul. 191.

onfôn = accipere, suscipere, sumere, percipere, empfangen, annehmen, vernehmen findet sich hier einmal mit sächlichem genitiv, während es sonst bei Cynewulf auch mit sächlichem dativ erscheint: pâm pe ic lifes onfonn lêohtes zelêafan Jul. 374.

strynan = accumulare, lucrari, acquirere wird mit dem gen. der zu erwerbenden sache konstruiert, das mittel der erwerbung steht im instr: se pe nu his feore nyle hôlo strŷnan Cr. 1574.

hlêotan = sortiri, nancisci mit gen. der sache, das mittel wird durch purh ausgedrückt: hôtað hỷ lầra lêana hlêotan purh wêpnes spor Jul. 622.

earnian = mereri, promereri mit sächlichem gen.: hû manna zehwilc Âr earnode êces lifes Cr. 1052; ze pæs earnedon Cr. 1350.

brûcsn = uti, frui, possidere, habere, gaudere, aliqua re mit sïchlichem gen.: môton ponne siððan sybbe brûcan êces êadwelan El. 1315; zeozuðe bracað and zodes miltsa Cr. 1686; yrfes brûcað .... wuldorcyninzes El. 1320.

Sehr oft findet sich auch brican mit der präposition mid verbunden, welche hier fur einen sociativen instrumental steht und nicht nur zur bezeiehnung einer begleitung von personen, sondern auch zur hervorhebung von begleitenden umständen verwendet wird: pæt he môze fore êzum eorðbâendra unscomiende êđles mid monnum brâcan bysmerlêas Cr. 1324 ; âwo tô ealdre enzla zemânan brâcà mid blisse Cr. 1647; ac pâr cyninzes żefe âwo brûcað êædizrs zedryht wuldres mid dryhten Cr. 1664; pæs ze fæzre sceolon lêan mid lêofum lanze brûcan Cr. 1361.

nêotan = frui mit sächlichem gen.: pæt pu môste zesêliz mines êðelríces êadiz nêotan Cr. 1461.

Neben dem genitiv findet sich bei nêotan such noch der instrumental, bei brican bisweilen der accusstiv. Im Gotischen ${ }^{\prime}$ finden wir bei brukjan = gebrauchen, neben dem genitiv oft den accusativ eines neutraladjectivums und auch den dativ; im Sanskrit folgt nach den verbis

1 Vgl. Schrader § 8. 
des 'geniessens, sich erfremens' der genitiv (auch acc.), der instrumental und local, im Grichischen nach $\chi \varrho \tilde{\eta} \sigma \vartheta \alpha \iota$ der dativ, im Lateinischen nach frui der ablativ (neben acc.) als vertreter des instrumental. Der von brukjan (brûcan) abhïngige genitiv berïhrt sich also mit dem instrumental; vielleicht diirfen wir ihn auch als vertreter desselben ansehen.

benêah (benuzan?) = fruor, potestatem habere mit sächlichem gen.: ponne he beza benêah El. 618 .

h) In gleicher weise wie die verba des 'geniessens' können wir die die verba des 'fiillens und siittigens' ' mit abhängigem genitiv als zu den faillen iiberleitend betrachten, in denen der genitiv einen anderen casus vertrat und neben diesem casus gebraucht wurde. Im Sanskrit werden diese verba sowol mit dem instrumental, als auch mit dem genitiv verwendet, im Lateinischen meist mit dem ablativ, der hier für den instrumental steht, seltener mit dem genitiv. Die gotischen hierher gehörigen verben sind sämmtlich schwache, von adjektiven abgeleitete und erscheinen fast nie in aktiver, sondern meist in passiver form mit dem genitiv verbunden. Im Ags. findet sich allerdings dann auch - und nachdem das gefuhh des vom verb abhängigen genitiv einmal lebendig geworden war, lïsst sich dies auch erklären - die aktive verwendung dieser verben mit genitiv, wie auch im Ags. der genitiv die anderen casus nicht verdrängte, sundern neben diesen gebraucht wurde. Von einer vollständigen vertretung des localis, ablativs und instrumentalis durch den genitiv in der weise, wie der dativ zum beispiel für diese casus eintrat, kann man also auch nicht sprechen, denn in allen den fällen, wo der genitiv an stelle eines friheren casus trat, findet sich stets noch ein dativ-instrumentalis, oder es wird das noch lebendige geflihl des ablativs durch präpositionen wie fram und of angedeutet.

Den grund für die vertretung des ablativs durch den genitiv findet Curtius $^{2}$ darin, dass er meint, im begriffe des ursprungs berïhren sich zusammengehürigkeit und das woher. Deutlicher wird dies noch, wenn man beriicksichtigt, dass sämmtliche mit dem genitiv verbundene verba den partitiven charakter tragen. Obgleich wir nun in dem begriffe 'partitiv' die teilung und trennung nicht empfinden und beim partit. genitiv in gegenteil an eine zusammengehörigkeit denken, so liegt diese doch zu grunde und mag wol auch hier den anknüpfungspunkt gegeben haben. Ganz falsch ist es allerdings, wenn man hier - wie es Nöldochen ${ }^{2}$ s. 27 und bei jedem anderen beispiele auch tut - diese trennung als bewegung von etwas fort für den woher-casus geltend machen will.

1 Vgl. Schrader $\$ 8$.

2 Curtius, Erläuterungen etc. s. 156.

3 Ueber den Gebrauch des Gen. im Mhd. Programm des gymnasiums zu Quedlinburg, 1868. Nöldechen tritt fir die lokaltheorie der casus ein und ist bemüht, für den genitiv im Mhd. das 'woher' durchzufthren. Er steht ganz auf dem standpunkte der lokalisten, indem er von dem casus, wie er uns erscheint, ausgeht und die etymologie des genitivs ganz unberiicksichtigt lïsst. Er verfährt auch insofern unhistorisch, als er das dem ablativ, instrumental und local zukommende nicht auscheidet, sondem alles zusammenbringt, was natïrlich - da er auf zweifelhafter grundlage aufbaut - auch zu schiefen resultaten fuihren muss. Den vom nomen 
Einer ursprünglichen berührung des genitivs mit dem ablativ steht auch syntaktisch nichts im wege, denn beide sind - natiirlich auf verschiedene weise - vom adjektiv gebildet', beide zeigen auch oft im Sanskrit gleiche endungen und fallen hier zuweilen zusammen.

âfyllan = implere hat den genitiv der sache, mit der man etwas anfuillt, bei sich: fŷres âfylled Cr. 1563. Die sache, die man mit etwas anfullt, steht im accusativ.

zefyllan = implere, füllen, anfüllen: forpon pu zefyldest foldan and rodoras .... wuldres pînes Cr. 408; pæt mon pæt lâmfæt lêades zefylde Jul. 577; swâ pu sylfa sîe synna zehwylcre firena zefylled Cr. 181; wuldres wæs zefylled cwêne willa El. 1135.

Wir sehen hier, dass von fünf beispielen zwei aktive verwendung zeigen, dass also das verbum schon vollstïndig als aktives transitives verb empfunden wurde.

Neben diesen verben, welche ursprïnglich einen genitiven gebrauch neben einem instrumentalen aufweisen, haben wir noch andere, bei denen der genitiv sich erweitert und funktionen von anderen casus, die dann verloren giengen, mit übernahm.2

2. Genitiv als vertreter des instrumentalis.

Neben dem dativ, den wir als eigentlichen vertreter des instrumentalis ansehen müssen, finden sich oftmals auch dieselben verben mit einem genitiv konstruiert.

ઉehlâdon = laden, congerere, imponere, cumulare: pæs pe on foldan in fyrndazum zôdes oðð̌ zâles zehlôd zeâra zonzum Cr. 1035.

hrê o $\mathrm{z}_{\mathrm{n}}=$ ornare, onerare hat den gegenstand, mit dem man schmiickt, im gen.: brŷd bêaza hrôden Cr. 292.

Bei cêspian = kaufen, erkaufen steht das erkaufte im gen., das mittel, wodurch man es erkauft, wird durch mid mit instr. und die person, für die man es erkauft, durch den dativ ausgedrückt: pôr he lêoflic liffes cêspode pêoden moncynne on pâm dæze mid phy weorde Cr. 1096.

zebß̊an = einen zu etwas bewegen oder zwingen. Hier steht der

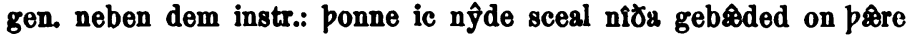
zrimmestan zodscyld wrecsn Jul. 203; pæt ic nŷde sceal nîða zebềed môd meldian Jul.462; pæt ic pisse nôte wæs nŷde zebæ̂ded Jul. 342.

zedreccan $=$ affligere, opprimere. Die sache, durch die man be-

abhängigen genitiv behandelt er nicht mit; dieser gerade wlirde ihn den genitiv als casus der zusammengehorigkeit und nicht als solchen der trennung gezeigt haben.

Vgl. Curtius, Chronologie s. 74 ff. u. 77.

2 Erdmann ( $\$ \$ 198,209,233,234$ ) leugnet zwar eine vertretung des ablativs und instrumentalis durch den genitiv, weil wir im Ahd. nie priipositionen, die dem ablativ zukommen, mit dem genitiv verbunden sehen, doch kann er sich in wirklichkeit dieser tatsache auch nicht entziehen. Wenn er sagt, der genitiv habe seine bedeutung von innen heraus so erweitert, dass der ablativ darin platz fand, so ist dies doch eine vertretung des ablativs durch den genitiv. Nur auf diese weise denke auch ich mir die übernahme von funktionen fremder casus durch den genitiv. 
driickt wird, steht in gen.: pêah hy him purh mînne noman wêrze wonhâle wæ̂tan bæ̂dan drynces zedreahte Cr. 1599.

3. Genitiv als vertreter des ablativs.

Zum grössten teile nahm allerdings der instrumental die last des ablativs auf sich und gab sie dann mit seinen eigenen funktionen an den dativ $a b$, doch findet sich auch bei den verben der trennung besonders eine vertretung durch den genitiv. Der grund dazu ist schon oben (s. 363) beriihrt worden.

âsceâdan = separare, segregare in reflexiver form mit dem genitiv der sache, von der man sich trennt': ac ic symle mec âscêd pâra scylda El. 470.

Mit dem genitiv der sache' ${ }^{\prime}$ wird auch âscy rian = destinare, separa.ce, sejungere, konstruiert; beide verba finden sich zusammen in: swâ bið pâra monna ælc âscyred and âsceâden scylda zehwylcre dêopra firena purh pæs dômes fŷ̀r El. 13 I2.

In diesem wie im folgenden beispiele steht purh zur bezeichnung des mittels der trennung.

â merian = excminare (de melallo liquefacto), purgare, merum reddere; zeclænsian = mundare, purgare;

zemyltan = liquefacere, emollire kommen zusammen mit dem gen. der sache, von der man sich trennt, vor: swâ bið pâra monna ælc âscyred and âsceâden scylda zehwylcre dêopra firena purh pæs dômes fŷr El. 1312; pret in wylme bið womma zehwylces purh ofenes fŷr eall zeclênsod, âmered and zemelted El. 1310.

biscerian, -irian, -yrian = privare, separare a re hat den gen. des beraubten gegenstandes nach sich: bescyrede enzla drêames Cr. 520.

bidælan $=$ expertum reddere, privare, sejungere wird ebenso konstruiert: Ic sceal feor panon hêanmôd hwêorfan hrôtra bidæled Jul. 681 .

In gleicher weise werden auch die folgenden verba gebraucht:

bilêosan = privare; noch mit dem dativ der beraubten person: swâ

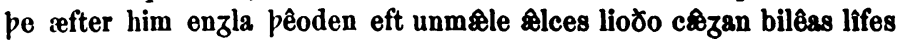
brytta Cr. 332.

birêafian = privare, spoliare, hat neben dem genitiv der beraubten sache noch den acc. der beraubten person: Hafað mec berêafod rihta zehwylces, feohzestrêona El.910; Hafað nu se hâlza helle berêafod ealles pæs folces Cr. 558 .

$\boldsymbol{\zeta}^{\mathrm{et}} \hat{\mathrm{x}} \mathrm{fan}=$ sejungere, separare, impedire, dirimere, detinere, privare: sundes zetwofde Cr. 987.

sceacan = moveri cum impelu, festinare, ruere, salire, volare, effugere steht mit dem gen. der sache, von der man sich entfernt: Bið se W (wên) scæcen eorðan frætwa Cr. 805.

of têon $=1$. detrahere, demere, entziehen: oft ic sŷne oftêob, ablende

1 Neben dem genitiv der sache findet sich auch als direkte fortsetzung der ablativen bedeutung bei ascyrian from und bei ascedden of und from mit dativ. 
beorna unrîm Jul. 468. 2. versagen, verweigern, vorenthalten; hier findet sich noch ein dativ der person und purh zur bezeichnung des mittels: and him 伊hwæs oftuzon purh heardne hyze hræzles nacedum Cr. 1505.

Hierzu kann man auch bepurfon = indigere, opus habere rechnen: wîsdômes bepearf, worda wærlîcra and wîtan snyttro El. 543; ponne ârna bipearf Jul. 715 .

Jeswican = intermittere, cessare, deficere, fallere, relinquere hat das, was man verschweigt, im gen. nach sich: zif we ..... pres unrihtes eft zeswîcað El.516; zif pu unrêdes ஷ̂r zeswîcast Jul. 120.

In ihrem gebrauche stehen zwischen altem instr. und ablativ (nach Schrader $\$ 8$ abl. causae) die verba der gemütsbewegung.

zefêohan, zefêon = laetari, delectari, gaudere, exultare mit dem gen. der sache, woruber man freude empfindet: hrefn weorces zefêah El. 110; cwên sî̀es zefêah El. 247; cwên weorces zefêah, on fyrhర్sefan El. 849; forpon we sculon ..... p pæs sêllran zefềon Cr. 757 .

zescomizan = erubescere mit dem gen. dessen, woruber man errötet: pæt ic yfeldæ̂da æ̂r zescomede Jul. 713; pæt hy bealudæ̂de âlces unryhtes êr zescomedon ..... earzra weorca Cr. 1303.

êadzan = beatificare zeigt einen dat. der person und den gen. der sache, durch die man beglückt wird: se pe êadzað us sizes Cr. 20.

4. Genitiv als vertreter eines alten localis.

Nur ein beispiel herrschen, walten über etwas lässt sich datür beibringen:

waldan = potestatem habere, possidere, dominare, gubernare, regere. Im folgenden beispiele ist neben dem gen. des herrschens noch ein acc. der zeit: walde wîdan fehro wuldres on heofonum, â bûtan ende, êcra zestealda El. 801.

In Juliana finden sich zwei heispiele, in denen bei waldan neben dem genitiv noch die präposition ofer gebraucht wird. Obgleich durch beide konstruktionen das 'herrschen ỉ ber etwas' bezeichnet wird, so decken sie sich doch nicht vollständig. Durch die präposition wird das rïumliche herrschen bezeichnet, während der genitiv bei der libertragung ('herr der siege sein') angewendet wird. Es scheint uberhaupt, als ob man beide konstruktionen (mit instr. und gen.) auf diese weise auseinander halten könnte (vgl. das vorige beispiel): se ofer mæzna zehwyle waldeð wîdeferh wuldres âzend, sizora zehwylces Jul. 222; pæt he sizora zehwæs ofer ealle zesceafte ânra wealde ..... êcra êadziefa Jul. 568.

5. Einmal findet sich auch ein genitiv absolut mit deutlich erkennbarer instrumentaler bedeutung gebraucht bei:

wiðscacan = repugnare, repudiare, abrenuntiare, abdicare (mit dat.

der person): wiðscæcest pu tô swî̀e sylfre ræ̂des pînum brydzuman Jul. 99. sylfre rêdes $=$ aus eigenem willen.

6. Zuletzt führe ich noch den genitiv bei wesan und weoryan an, den man (nach Grimm IV, 652) 'den pridikativen nennen dïrfte, weil er sich leicht in ein substantives oder objektives prädikat auflösen lïsst:

wesan: He is for eorðan æðeles cynnes, wordcræftes wis and witzan 
sunu, bald on meðle El. 591; ponne pæes tîd ne bið Cr. 1567; pone lytlan frist, pe her liffes sŷ Cr. 1323; pâra on hâde sint ..... syx zenemned El. 740; pâra sint fêower El. 744; Sum wæs êhtwêliz æð̌les cynnes rîce zerêfa Jul. 18; næs pâ friczendra.... zầfeorran zeferede El. 991; hwæt pæes wæ̂re dryhtnes willa El. 1160.

weorðan: weorठan and pæs in life lize ne wyrðeð El. 575.

7. In vielen fällen kann man den häufig vorkommenden genitiv pass mit 'dafitr, deswegen, weil' iibersetzen; es lässt sich hier meist nicht genau entscheiden, ob der genitiv zum nomen oder zum ganzen satze gehört: næs pæs æniz môdiz man ofer eorðan purh hâlze meoht Jul. 513.

poes mit dat. pers.:

pyncean: nu ze zeare cunnen, hwæt êow pæs on sefan sêlest pynce tôzyðannne El. 531; hwæt him pæs on sefan sêlost pâhte tô zefâstenne El. 1165. - wesan: him bið enzls weard milde and blî̀e, pæs El. 1316; sîe pe mæzena zod prymsittendum panc bûtan ende, pæs pu El. 810; him wæs zêomor sofa, pæs Cr.499. - zelimpan: pæs hire se willa zelamp purh bearn zodes beza zehwæðeres El. 963. pancjan: zode pancode sizora dryhtne, pæs pe El. 1139 .

poes mit acc. rei:

そe そearwian: nêfre pu pæs swî̋lîc swâ zezearwast purh hểstne nî̀ heardra wîta Jul. 55; ne wîta pæs fela zezearwast Jul. 177. - zemunian: pæs he eftlêan wille purh eorneste ealles zemunian Cr. 1110. - wîtan: pæs pu ponc ne wisses Cr. 1386. - talizæn: pæs ic sổ talze Cr. 794. - anforlêtan: pæs hy swâ fæzre zefêan on fyrndazum and swâ æ̂nlîce ânforlêtun Cr. 1295. - onfôn: pæs hi lonze sculon ferðwêrize onfôn in fŷrbâte .... wrâtlic andlêsn Cr. 530. - âzan: wuldor pæs âze on hêahnesse heofonrices zod El. 1124; wuldor pæes âze prŷnesse ponc butan ende Cr.598. âdrêozan: pæs ze sceolon hearde âdrêozan wite tô wídan ealdre Cr. 1514. - prôwjan: pæs he in êrmơum scesl eslra fulla ful fâh prôwjân, pêownêd pôlian El. 770. - zesêon: pæs po hîe fêonda zefâr fyrmest zeŝिzon El. 68. - forsêon: pæs po hie mâna zehwylc forsâwon. - brûcan: pæs ze fæzre sceolan lêsn mid lêofum lanze bracan Cr. 1361. - wealdan: pæs ठu, zod dryhton, wealdest widan ferhð El. 670.

poes mit dat. pers. und acc. rei:

seçan: ponc seczan - we pæs ponc mâzon seczan sizedryhtno El.12i; we pæs ealles sculon seczan ponc and lof dryhtne ussum and hûru pêre hæ̂lo Cr. 611. - lèan zifan: ne him fæzre pæs lêofum zesî̀rum lêan æfter zeaf Cr. 472. - pæs he earfeðu polado lêodum tô helpe Cr. 1174.

paes mit instr.:

lifan: beorht cyninz lêanè, p’es pe hy on eoròan earzum dâdum lifdon leohtrum fâ Jul. 829. - brêcan: pæs ic lustum brêac willum in worulde El. 1251.

paes mit sächl. dat. und instr.:

healdan: pæs pe hi hyra pêodnes wel wordum and weorcum willum beoldon Cr. 1236. 


\section{III. \\ Der genitiv nach adjektiven.}

§ 18. Auch hier muss man unterscheiden zwischen denen, welche ursprtinglich mit einem genitiv verbunden waren und denen, die in ihrem gebrauche auch noch mit dem dativ als vertreter von ablativ und instrumentalis verbunden sind. Dies sind die adjectiva, welche fulle und $m$ angel bezeichnen, sowie schuldig, froh, stolz, gesund, stark, kuhn.

Ich folge hier ganz Nader's ${ }^{1}$ anordnung und stelle voran die adjectiva, welche fülle und mangel bezeichnen:

ful: wuldres ful El. 752; ealra fûla .. El. 769; wîsdômes .. El. 939; sizores .. Cr. 88; weorðmynda .. Cr. 378; beorhtblæ̂des .. Cr. 1658; full hâlzan hyhtes Cr. 57; cearena .. Cr. 962; leahtra .. Jul. 612; ceazealdra .. Jul. 618; ældes fulle Cr. 960; fŷres .. Cr. 1626; sâres fulne Cr. 1517; ezsan fulne Cr. 1370. - 叉̂htspêdiz: æxhtspedizra feohzestreona Jul. 101. - lêas: eallra znyrna lêas El. 422; synna lêase Jul. 188. 614, Cr. 1641; leohtra .. Jul. 566. 583; hyhta .. Jul. 652; duzữa lêas El. 693; duzuð̌a lêase Cr. 1509; mânes lêas Cr. 36; firena lêas Cr. 123; helpendra .. Cr. 1414; womma .. Cr. 1452. 1465; womma lêase Cr. 188; synna lêasne El. 197. 778; dômes .. El. 945. - ze witlêss: pæs zewitlêas Cr. 1473. - zêas: zôda zêasne El. 924,

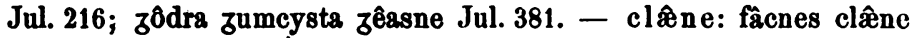
Jul. 565; mâna zehwylces .. Jul. 30; womma . . Cr. 1694 . - orwêna: friò orwêna Jul. 320. - tôm: manweorca tôme Cr. 1211; tîres wone Cr. 270; hræzles nacedum Cr. 1505.

Bereitschaft, lassigkeit:

fûs: sîঠes fâs El. 1219. - zearo: zearo sôna unwâclíce willan pînes Jul. 49. - s ̊̂ne: pæs sîðfætes sæ̂ne El. 226.

Wert, schuldig:

wyrde: pæt is pæs wyrðe Jul. 103. 643, Cr. 600; weorðe worda and dêda peawa and zeponcs Cr. 1583. - unwyrðe: feores unwyrðe Cr. 1563. - unscyldiz: unscyldigne eafota zehwylces El. 423.

Eingedenk:

zemyndiz: zemyndiz peodnes willan El. 266; .. dryhtnes willan Jul. 601; minra zylta .. El. 817; yfela . . El. 902; eafoða . . Jul. 601; tynna .. El. 940. - andwis: yfeles andwis Jul. 244. -- wîs: worderæftes wis El.592. - zearosn ottor: zidda zearosnottor El.418, Cr.713. zlêsw: bôca zlêsw El. 1212.

Froh, stolz, gesund, stark, kühn:

cræftiz: wordes cræftiz El. 314. 419; pæs hyzecræftiz Cr. 241. - ân-

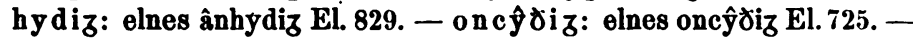
zolds pêdiz: pæ̂re wifżifta zoldspêdiz Jul. 38. - ôrwî̉z: æxzwhæs ôrwizne Jul. 434. - pweorht $\hat{y} m e$ e: pweorhtimbran mæzða cynnes Jul. 550. - zno rn: znorne .. dômes pæs miclan El. 1205.

1 Nader a. a. o. $\$ 24$. 
IV.

\section{Adverbialer genitiv.}

$\S 19$. Wir finden im Angelsächsischen sehr hänfig genitive von substantiven, adjektiven und pronomen in adverbialer verwendung, welche nicht nur dem verbum, sondern oft auch dem ganzen satze lokale, temporale oder modale bestimmungen hinzuftgen. 1

Lokaler genitiv.

Nur einen fall kann ich aufzeichnen, in dem ein substantiv im genitiv lokale bedeutung hat: and in pêostrum her sâton sinneahtes synnum bifealdne Cr. 117.

Temporaler genitiv.

Dazzes und nihles findet sich zuweilen in adverbialer verwendung:

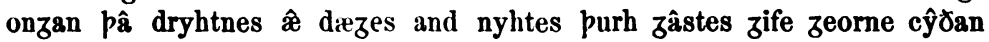
El. 198; pe âr moncynne nihtes lŷhte Cr. 939 .

Ihrer form und bedeutung nach sind folgende genitive vollständig zu adverbien geworden: sinzales, pl. sinzala = bestïndig; unzêara = neulich, bald; semninza = alsbald; fârinza = plötzlich; zènunza $=$ geradezu, vollständig.

sinzales: and hio ponne æfter him êce stondað simle sinzales swâ beclŷsed Cr. 323 ; pæet hy môtan his ætwiste eazum brûcan simle sinzales swezle zehyrste weorðian waldend wîde and sîde Cr. 393. unzêara: pæt pu unzêara ealdre scyldiz purh dêora zripe deaðe sweltest Jul. 124. - semninza: pâ wear hlûd zehyred Cr. 491; on swearte niht sorzlease hæleð semninza forfêhð slæ̂pe zebundene Cr. 874; ponne semninza on Sŷne beorh sîðan-eastan sunnan leoma cymeð of scyppende scŷnan leohtor Cr. 900; pâ cwom semninza in pæt hlinreced hæleða zewinna yfeles andwis Jul. 242; pâ cwôm semninz̊ heßn helle-zæest Jul. 614; бâ cwom semninza sunnan beorhtra lâcende lìz El. 1110; and eft semninza swîze zewyrðe $\delta$ in nêdclofan nearwe zeheaðrod El. 127.. そezninza: pâ scealt zeazninza wîsdôm onwrêon El. 673. - færinza: pæt hi færinza feorh âlêton purh ædra wylm Jul. 477; pæt hi fârinza ealde rfponcan edniwedan beore druncene Jul. 484.

Hierzu fuige ich noch einige adverbien, welche 'allgemein das gebiet der tätigkeit des verbs bestimmen oder dem ganzen satze eine allgemeine bestimmmung verleihen. ${ }^{2}$

elles: ne mæz he elles brinzan tô bolde Jul. 113. - eallinza: pæt se him eallunza owiht ne ondrâdeð Cr. 923. - edniowînza: fram blindnesse bôte zefremede ednîowunza purh pæt æð̌ele spâld El.300. undearninza: Elene mapelade and for eorlum spræc undearninza El. 405; Him pâ sèo êadize andwyrde âzeaf Elene for eorlum un-

1 Vgl. Koch, Historische Grammatik der englischen Sprache, band II, herausgegeben von Julius Zupitza. S. 311).

2 Vgl. Nader $\$ 23$; Erdmann II, 215. 
dearnunza El.620. - sôna: and sôna forlæt weall wið wealle Cr. 10; sôna wôron zearwe hæleð mid hlâford tô pêre hâlzan byriz Cr. 460; and pâ sona zelomp Cr. 233; w⿳⺈冂on Romware, seczas sizerôfe, sôna そezearwod wæ̂pnum tô wîzze El. 47; he wæs sôna zearu El. 85; ac wæs sôna zearu wîf on wilsî̀ El. 222; zif we sôna eft pâra bealudæ̂da bôte zefremmap El. 514; hîe pæt ôfstlîce efnedon sôna El. i13; he sôna âras zâste zezearwad El. 888; hîe sôna pâr purh pâ hâlzan zescerft helpe findap El. 1031. - micles: tô late micles Jul. 444. lytes nâ: wæs his rîce brâd, wîd and weorðlîc ofer werpeoda,

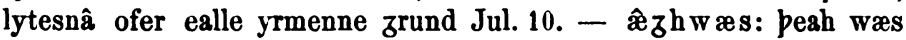
hyre mæzdenhâd æ̧̂̉hwæs onwalł Cr. 1421; pæt pu mec pus fæste fetrum zebunde æ̂zhwæs orwîzne Jul. 434; Is an me sweotul, pæt pu unscamze æ̂zhwæs wurde on ferðe frôd Jul. 552; Heo inlîze stôd æzhwæs onsund Jul.593. - nalles: nalles for torne El.1134; pâra pe ic zefremede nalles fêam sîðum El. 823. - nales: nales fore lytlum Cr. 963; hwâ hy mid blêdum sceôp, monze nales fea Cr. 1171; hâlze hyzezlêawe hæleðum sæżon oft nales æ̂ne ymb pæt æðele bearn Cr. 1195; nales dryhtnes zemynd siððan zesêcað Cr. 1537; on him dryhten zesihð nales feara sum firenbealu lâđlîc Cr. 1276; nales frætwe onhêht Jul. 118; nales znyrnwræ̂cum feozað frŷnd hiera El. 359; Ic pæs wuldres trêowes oft, nales æ̂ne, hæfde inzemynd El. 1253; pârః pe ic zefremede nalæs feam sîðum Jul. 354 .

Auf die kausale bedeutung des in vielen fällen adverbiell gewordenen genitiv ist schon hingewiesen worden.

Zweimal findet sich auch in Crist der adverbial gewordene genitiv

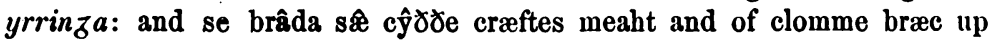
yrrinza on eorðan fæðm Cr. 1147; ponne he yrrinza on pæt fræte folc firene stôleð lâðum wordum Cr. 1373. position.

§ 20. Der genitiv beim lokaladverb und bei der prä-

Nur wenige beispiele lassen sich hierfür beibringen.

Lokaladverbien:

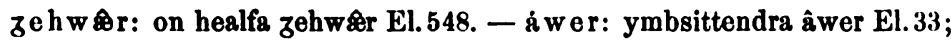
ôwêr æ̂nzes Cr. 199; londes .. Cr. 1002.

Von präpositionen findet sich nur $t \hat{b}$, welches zuweilen mit dem genitiv verbunden ist: Is pes hæet tố đan stranz, prêanŷd pæs pearl and pes proht tô đæs heard dôzorrîmum El. 703; Nis âniz nu eorl under lyfte secz searoponcol tô pæs swî̀te zlêaw Cr. 220.

Dann findet sich noch in folgenden stellen tózeznes (tozednes, tozềnes): El. 356. 167, Cr. 546. 548. 575.

BERNBURG.

R. RŐSSGER. 